\title{
Projection Kinematic Analysis of DNA Origami Mechanisms Based on a Two-Dimensional TEM Image
}

\author{
Lifeng Zhou, Hai-Jun Su', Alexander E. Marras, Chao-Min Huang, and Carlos E. Castro
}

\begin{abstract}
In this paper, a new method called "projection kinematics" is presented for the determination of 3D configurations of DNA Origami Mechanisms (DOM) based on a single 2D projected image. The method enables finding all possible projected configurations of a DOM in space by solving the projection kinematics equations based on the minimum needed information measured from a $2 \mathrm{D}$ image. If redundant measurements are available, they can be exploited to eliminate ambiguous solutions. First, projection kinematic analyses were derived for the basic kinematic joints - revolute, prismatic, cylindrical and spherical joints and one degree-of-freedom (DOF) planar four-bar and two DOFs five-bar linkages. Then a generalized procedure for projection kinematic analysis is presented. Finally, a universal joint and a Bennett linkage fabricated by DNA origami self-assembly are chosen as proof-of-concept examples to verify the feasibility of projection kinematic analysis of DOM. This approach not only provides valuable guidance for the design of DOM, but also offers a low cost analysis approach for configuration estimation of spatial mechanisms when images from multiple viewing angles are not accessible, which is generally the case for the most commonly used analysis methods.
\end{abstract}

Keywords: Projection kinematics, configuration analysis, DNA origami mechanisms, DNA nanotechnology

\begin{abstract}
Abbreviations
DNA, Deoxyribonucleic acid; DOM, DNA origami mechanisms; 2D, two-dimensional; 3D, threedimensional; EM, Electron microscopy; TEM, Transmission electron microscopy; AFM, Atomic force microscopy; DOF, degree-of-freedom; Cryo-EM, Cryo-electron microscopy;
\end{abstract}

\section{Nomenclature}

$\theta_{0 i} \quad$ Initial configuration joint angle

$\theta_{i} \quad$ True transformed joint angle

$\bar{\theta}_{i} \quad$ Projected or measured joint angle

$\bar{\theta}_{i-P} \quad$ Projected joint angle obtained from the projection kinematics analysis

$L_{i} \quad$ True link length 
$\bar{L}_{i} \quad$ Projected or measured link length

$\bar{L}_{i-P} \quad$ Projected link length obtained from the projection kinematics analysis

A $\quad=(x, y, z)^{\mathrm{T}}$, bold faced letter represents a 3 by 1 vector

$\overline{\mathbf{A}} \quad=(x, y, 0)^{\mathrm{T}}$, a vector projected to the $x y$ plane

$[X(\bullet)] 3$ by 3 rotation matrix about $x$-axis

$[Y(\bullet)] 3$ by 3 rotation matrix about $y$-axis

$[Z(\bullet)] \quad 3$ by 3 rotation matrix about $z$-axis

[R] A general 3 by 3 rotation matrix

[P] The projection matrix to the $x y$ plane

\section{Introduction}

Structural DNA nanotechnology enables the construction of complex geometrical nanostructures through the bottom-up self-assembly of single DNA strands based on the Watson-Crick base pairing principle [13]. DNA origami structure typically consist of bundles of double-stranded DNA where the cross-sectional dimensions are a fuction of the arrangement and diameter of DNA helices, about $2 \mathrm{~nm}$, and the length of each component can be deteremined by the base count and the length of each base pair, which is about $0.34 \mathrm{~nm}$ quantified experimentally [4,5]. Recent advanced DNA nanotechnologies, including scaffolded DNA origami [4-6], have promoted and expanded the design of dynamic DNA nanostructures.

We previously demonstrated the ability to use scaffolded DNA origami to design and fabricate a series of nanoscale classic kinematic joints, such as the revolute, prismatic, and universal joints as well as kinematic mechanisms including a spatial four-bar Bennett linkage, a crank-slider mechanism and a scissor mechanism [7-9]. In addition, we implemented the design methodology of compliant mechanisms to construct a compliant hinge joint and a four-bar bistable mechanism [10-12]. We call these nanostructures DNA Origami Mechanisms (DOM). By mimicking concepts of links and joints in macroscopic mechanisms, we demonstrated that these DOM can produce a prescribed 1D, 2D, or 3D motion pattern. While important progress has been made towards design, fabrication and actuation of DOM, quantitative validation of their kinematic motion remains a challenge. Currently, design validation analysis of these DOM relies on measurement of projected geometric parameters, such as link lengths or angles between two links [7,13], measured from Transmission Electron Microscopy (TEM) or Atomic Force Microscopy (AFM) images. TEM provides a 2D projection of 3D structures, and AFM images give height information, but only of the upper most surface of a structure. Hence, these images do not represent the true kinematic parameters in space and it is difficult to fully prescribe the configurations of DOM, especially ones with 3D motion.

3D reconstruction provides a powerful technique that can obtain detailed structure information from one or multiple 2D images of many or even a single copy of a structure or mechanism, such as the facial and terrestrial reconstructions [14-17]. Nevertheless, they are highly challenging, time consuming, and often have to average over many images from various viewing angles. Hence, they are not effective for estimating spatial mechanisms with one or more degrees-of-freedom from a single 2D image. Electron microscopy-based reconstruction techniques provide a potent technique to determine the 3D geometry of 
biomolecules with nanometer or subnanometer resolution by taking a series of images from different view angles [18-20]. However, this technique depends heavily on the preparation quality of the sample and often requires averaging over many structures, which is problematic for DOM with mobility greater than one. In addition, the post imaging-processing algorithm for 3D reconstruction from 2D TEM images remains highly challenging.

Here we present an alternative approach to validate the kinematic motion of DOM by analyzing its projected kinematic constraint equations to determine the relative spatial geometries of links projected on a 2D plane. Intuitively, the projected configuration of a DOM is determined by its configuration in space as well as the projection direction. If the projection direction is fixed, the change of projected configuration will follow the motion of the DOM in space. For example, Su et al. [13] first derived the motion of the Bennett linkage in space and then projected the entire Bennet linkage configuration on a designated 2D plane. By kinematic analysis of a one DOF Bennett 4-bar linkage, Su et al. successfully determined the projected joint angles and compared them with measured data from 2D TEM images.

In this paper, we expand this method to the kinematic analysis of projected configurations of general mechanisms. We call this approach "projection kinematics." It differs from the projective kinematics in that projective kinematics reconstructs a 3D object from at least two stereo images [21] while our projection kinematics predicts 3D configurations using one 2D image. In addition, traditional kinematic analysis deals with motion using true kinematic parameters, while projection kinematics is based on the projected parameters. Also, in contrast to $3 \mathrm{D}$ reconstruction from $2 \mathrm{D}$ images used in machine vision that usually focuses on single static object [22,23], projection kinematics analysis predicts the actual configuration of a multibody mechanism based on the information extracted from a single projected 2D image.

This paper is organized as the following. First, a general algorithm of the projection kinematics analysis for mechanisms such as four-bar and five bar linkages is derived based on the projection kinematics of various kinematic joints. Second, a universal joint and Bennett linkage fabricated by DNA origami are used as two case studies to demonstrate the feasibility and describe the application process of projection kinematics. Multiple solutions can be obtained by solving the projection kinematics equations based on the variables measured from projected configuration. Also, different measurements, such as the combination of different angles or link lengths can result in different projection kinematics equations, which can obtain different solutions with some of them being mechanically distinct. Usually, measuring angles between two links is easier and more accurate than measuring lengths because the ends of DNA origami links are often blurry in TEM images, especially for biological materials that are generally flexible and do not give clear contrast. Lastly, we discuss the minimum number of variables that must be measured for solving the projection kinematics equations and how to determine the correct solution for the spatial configuration out of the multiple possible solutions..

\section{Projection analysis of kinematic joints}

The configuration of a DOM is determined if it is fully controlled, i.e. the number of actuated kinematic parameters equals the number of degrees-of-freedom (DOF). Then the entire mechanism can be treated as a static structure and its configuration in space can be modeled by conducting the transformation in a global frame. For convenience, we define the project direction as the $z$-axis. The projection plane is the $x y$ plane. Only rotations about $x$ and $y$ axes alter the projected configuration of the DOM. In addition, rotation about the $x$-axis does not affect the projected length of a link placed along the $x$-axis, similarly for the $y$-axis.

Mathematically, the rotation matrices about the $x$-axis (rotation angle $\alpha$ ) and $y$-axis (rotation angle $\beta$ ) are defined as 


$$
[X(\alpha)]=\left[\begin{array}{ccc}
1 & 0 & 0 \\
0 & \cos \alpha & -\sin \alpha \\
0 & \sin \alpha & \cos \alpha
\end{array}\right] \text { and }[Y(\beta)]=\left[\begin{array}{ccc}
\cos \beta & 0 & \sin \beta \\
0 & 1 & 0 \\
-\sin \beta & 0 & \cos \beta
\end{array}\right]
$$

Combining these gives the resultant rotation matrix $[R]$ as

$$
[R]=[Y(\beta)][X(\alpha)]=\left[\begin{array}{ccc}
\cos \beta & \sin \alpha \sin \beta & \cos \alpha \sin \beta \\
0 & \cos \alpha & -\sin \alpha \\
-\sin \beta & \sin \alpha \cos \beta & \cos \alpha \cos \beta
\end{array}\right]
$$

For any given joint, or mechanism, the projection kinematics analysis will determine all possible spatial configurations that can have a particular projected configuration. Because of the practical difficulties in measuring the detailed geometric shape of a link from a 2D image, we simplify a link into a single line, which is sufficient to reconstruct the kinematic configuration of the DOM.

Derivation of projection kinematics for mechanisms is based on the projection analysis of classic kinematic joints. The top of Fig. 1 shows sketches of revolute, prismatic, cylindrical and spherical joints in $3 \mathrm{D}$ space and their projected configurations on the $x y$ plane are shown at the bottom of Fig. 1. Origin of the global coordinate frame is placed at the point $\mathbf{O}$ and $x$-axis is pointing in the direction of link $O A$ and $z$-axis is the projection direction.
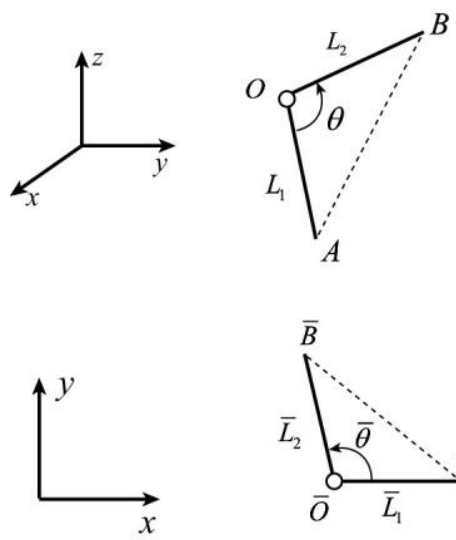
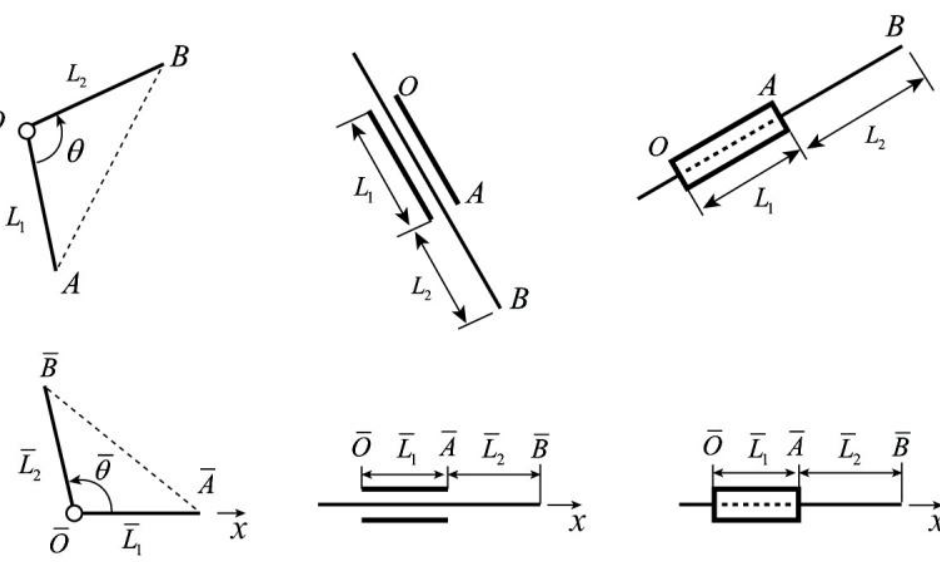
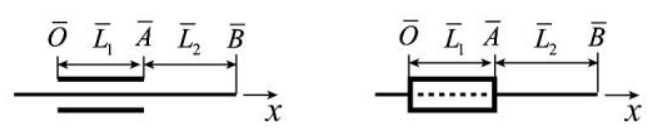
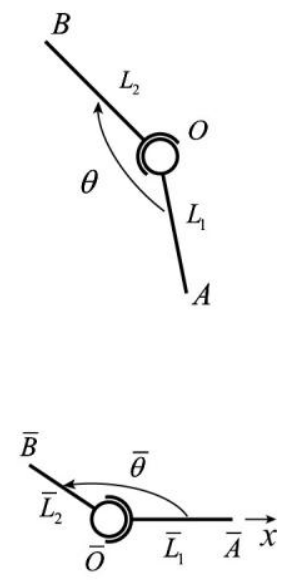

Fig. 1. Kinematic parameters of revolute, prismatic, cylindrical and spherical joints (from left to right) are 3D (top) in nature and they are projected to the $x y$ plane (bottom).

In the following sections, the projection kinematics of the revolute, prismatic, cylindrical and spherical joints are discussed in detail.

\subsection{Revolute joints}

A revolute joint is constructed by two links connected with a pin joint to yield one rotational DOF. Fig. 2 shows a sketch of a revolute joint, where cylinders represent links and balls represent joints and end points of links. Note this representation method, which neglects the shape of links and joints, is also used in the following sections. 

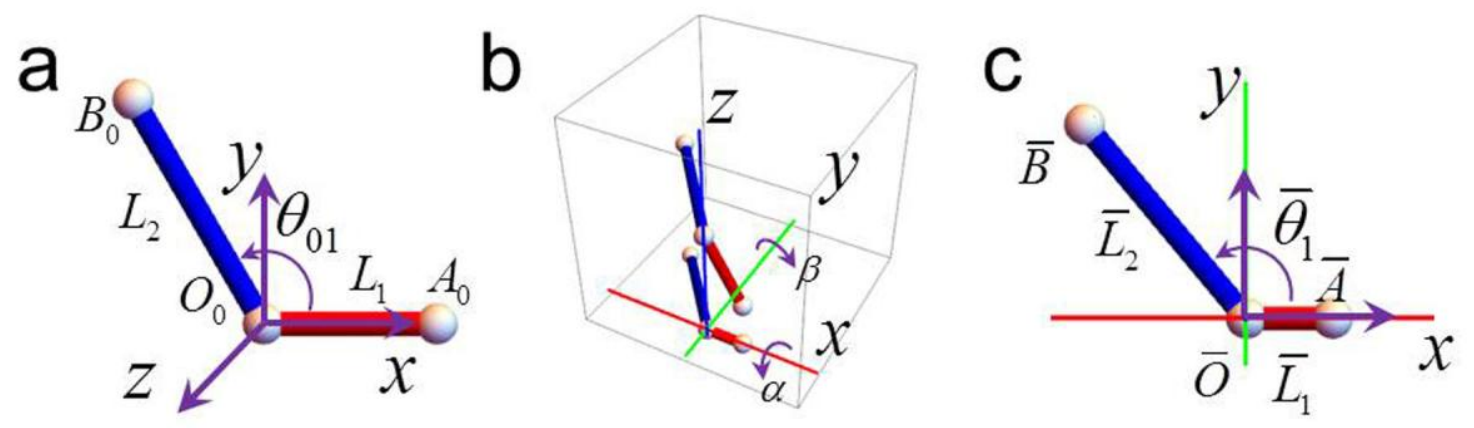

Fig. 2. Projection of a revolute joint. (a) Local coordinate frame $x y z \mathrm{O}_{0}$ is defined with $x$-axis along one link and $x y$ plane on the plane formed by the two links, (b) 3D projection (in order to clearly show the spatial and projected configurations respectively, the spatial configuration is shifted vertically to a position above the $x y$ plane), (c) projected configuration.

Here, we assume that both links are initially laid flat on the $x y$ plane with the link $\mathbf{O}_{0} \mathbf{A}_{0}$ along the $x$-axis and $\mathbf{O}_{0}$ at the origin position of the global frame, as shown in Fig. 2a. Here the two link lengths are $L_{1}$ and $L_{2}$, and the joint angle is $\theta_{01}$.

For convenience, let us define the $x y$ plane projection matrix as

$$
[P]=\left[\begin{array}{lll}
1 & 0 & 0 \\
0 & 1 & 0 \\
0 & 0 & 0
\end{array}\right]
$$

By multiplying $[P]$, projection coordinate $(x, y, 0)$ on the $x y$ plane of any spatial point $(x, y, z)$ can be obtained. Geometric information for the projected configuration (Fig. 2c), namely the projected link lengths $\bar{L}_{1}, \bar{L}_{2}$ and the projected angle $\bar{\theta}_{1}$ can be measured by image analysis tools.

Table 1 Initial, rotated, projected and measured points that define the configuration of the revolute joint

\begin{tabular}{|l|l|l|l|}
\hline Points in joint reference frame & Points after rotation & Projected points & $\begin{array}{l}\text { Measured points from } \\
\text { projected 2D image }\end{array}$ \\
\hline $\mathbf{A}_{0}=\left(L_{1}, 0,0\right)^{T}$ & $\mathbf{A}=[R] \mathbf{A}_{0}$ & $\overline{\mathbf{A}}=[P] \mathbf{A}$ & $\overline{\mathbf{A}}=\left(\bar{L}_{1}, 0,0\right)^{T}$ \\
\hline $\mathbf{B}_{0}=\left(L_{2} \cos \theta_{01}, L_{2} \sin \theta_{01}, 0\right)^{T}$ & $\mathbf{B}=[R] \mathbf{B}_{0}$ & $\overline{\mathbf{B}}=[P] \mathbf{B}$ & $\overline{\mathbf{B}}=\left(\bar{L}_{2} \cos \bar{\theta}_{1}, \bar{L}_{2} \sin \bar{\theta}_{1}, 0\right)^{T}$ \\
\hline
\end{tabular}

Comparing the coordinates of projected and measured points in Table 1, we can obtain

$$
\left\{\begin{array}{l}
L_{1} \cos \beta=\bar{L}_{1} \\
L_{2}\left(\cos \theta_{01} \cos \beta+\sin \theta_{01} \sin \alpha \sin \beta\right)=\bar{L}_{2} \cos \bar{\theta}_{1} \\
L_{2} \sin \theta_{01} \cos \alpha=\bar{L}_{2} \sin \bar{\theta}_{1}
\end{array}\right.
$$

Parameters $\alpha, \beta$ and $\theta_{01}$ can be solved from Eq.(4) when $\bar{L}_{1}, \bar{L}_{2}$ and $\bar{\theta}_{1}$ are measured from the projected configuration. It is noted that only the last two equations in Eq.(4) are mildly coupled. Solving the first equation yields two solutions of $\beta$. Substituting either of those solutions for $\beta$ into the last two equations in Eq.(4) and eliminating $\alpha$ leads to an equation for $\theta_{01}$, which we can be solved to obtain 
four solutions. Hence, we conclude that there are at most eight solutions for the analysis given by Eq.(4).

A numerical example is shown below to validate the projection kinematics solution process for a revolute joint. First, the length of the links, the joint angle $\theta_{01}$ and global rotation angles $\alpha, \beta$ are chosen randomly (excluding extreme positions, such as the link perpendicular to the $x y$ plane) and shown in the first row of Table 1. Second, the designed projected configuration can be obtained and the projection variables are shown in the third row of Table 2.

Table 2 Numerical example for projection kinematics analysis of the revolute joint

\begin{tabular}{|c|c|}
\hline Initial configuration & $L_{1}=100, L_{2}=150, \theta_{01}=120^{\circ}$ \\
\hline Rotation angles & $\alpha=-30^{\circ}, \beta=60^{\circ}$ \\
\hline Projected configuration & $\bar{L}_{1}=50, \bar{L}_{2}=195.256, \bar{\theta}_{1}=129.809^{\circ}$ \\
\hline
\end{tabular}

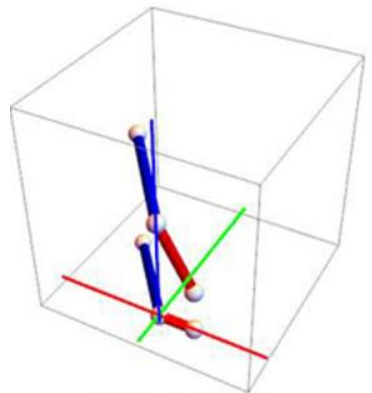

$\left(120^{\circ},-30^{\circ}, 60^{\circ}\right)$

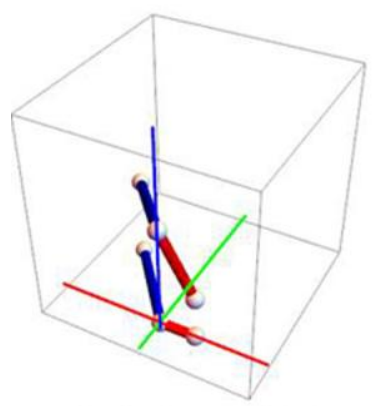

$\left(97.18^{\circ},-40.89^{\circ}, 60^{\circ}\right)$

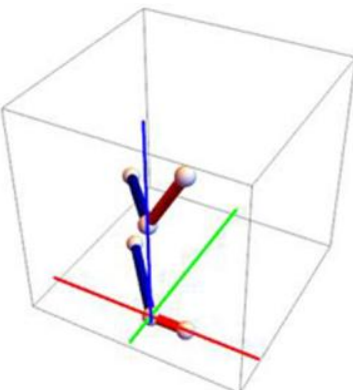

$\left(120^{\circ}, 30^{\circ},-60^{\circ}\right)$

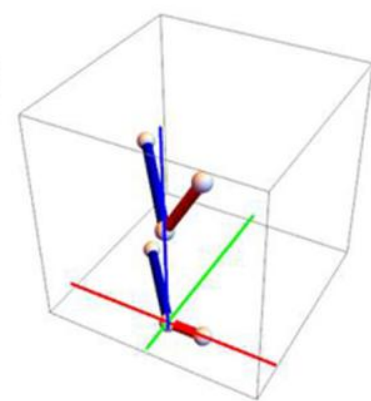

$\left(97.18^{\circ}, 40.89^{\circ},-60^{\circ}\right)$

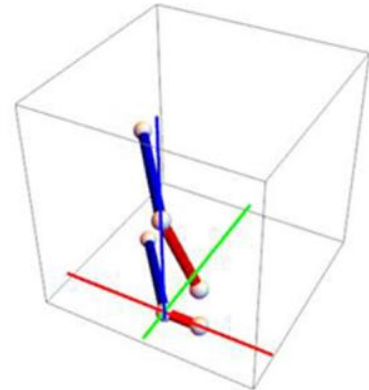

$\left(-120^{\circ}, 150^{\circ}, 60^{\circ}\right)$

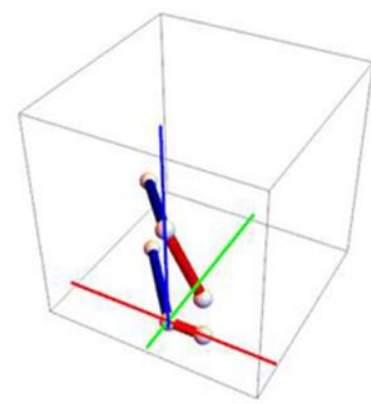

$\left(-97.18^{\circ}, 139.11^{\circ}, 60^{\circ}\right)$

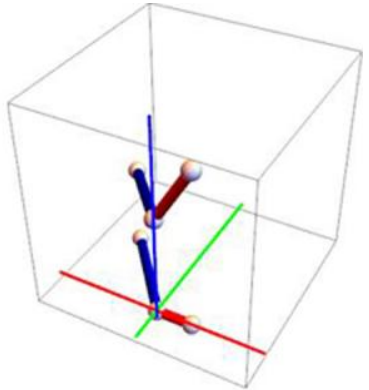

$\left(-120^{\circ},-150^{\circ},-60^{\circ}\right)$

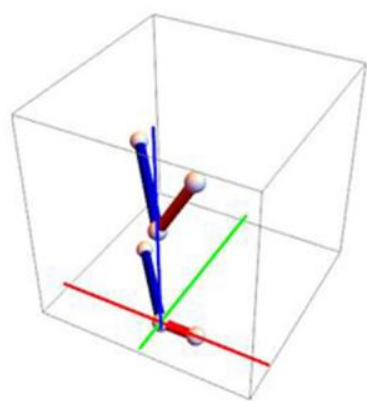

$\left(-97.18^{\circ},-139.11^{\circ},-60^{\circ}\right)$

Fig. 3. Configuration solutions of the projection kinematics analysis of a revolute joint, $\theta_{01}, \alpha$ and $\beta$ for each solution are shown under each configuration as $\left(\theta_{01}, \alpha, \beta\right)$.

Eight solutions are obtained by solving Eq. (4) when keeping $\theta_{01}, \alpha$ and $\beta$ as unknowns and substituting the other variables shown in Table 1 into the equations. Fig. 3 shows the eight possible solutions that can have the same projected configuration, where each solution has the spatial configuration above the $x y$ plane and the projected configuration on the $x y$ plane. The configuration determined by the first solution is the same as the specified initial configuration shown in Table 2 . The second solution has the same joint angle as the designed, but the global rotation angles $\alpha$ and $\beta$ are actually opposite as designed. This means the revolute joint has two spatial positions that can have the same projected configuration. These kinds of solutions can be grouped as a set. Then, the third and fourth solutions construct another set whose joint angle is $-120^{\circ}$ which means the joint rotates in the opposite direction. In addition, $\alpha$ of the third and fourth solutions has the negative supplementary angle values of the first and second solutions respectively, while $\beta$ is the same. If the rotation direction can be determined from the projected configuration, for example by 
some asymmetric feature on a link, then the solutions with opposite rotation angle can be eliminated. The absolute angle value of the bottom four solutions is $97.18^{\circ}$, which means the revolute joint can have two different joint angles that correspond to the same projected configuration. Fig. 4 shows two examples of symmetrical (or mirrored) configurations of a revolute joint (Fig. 4a) and planar four-bar linkage (Fig. 4b). An asymmetric feature, indicated by the small red box placed on the purple links can specify the rotation direction of the revolute joint and also differentiate the symmetrical configurations of the four-bar linkage. This provides an important criterion for design of DOM if 2D images are to be used for the analysis and verification of motion. Alternatively, if the motion can be constrained by design, for example to be between $0^{\circ}$ and $180^{\circ}$, then the joint angle can be uniquely specified. We previously used this approach for a DNA origami hinge [7].

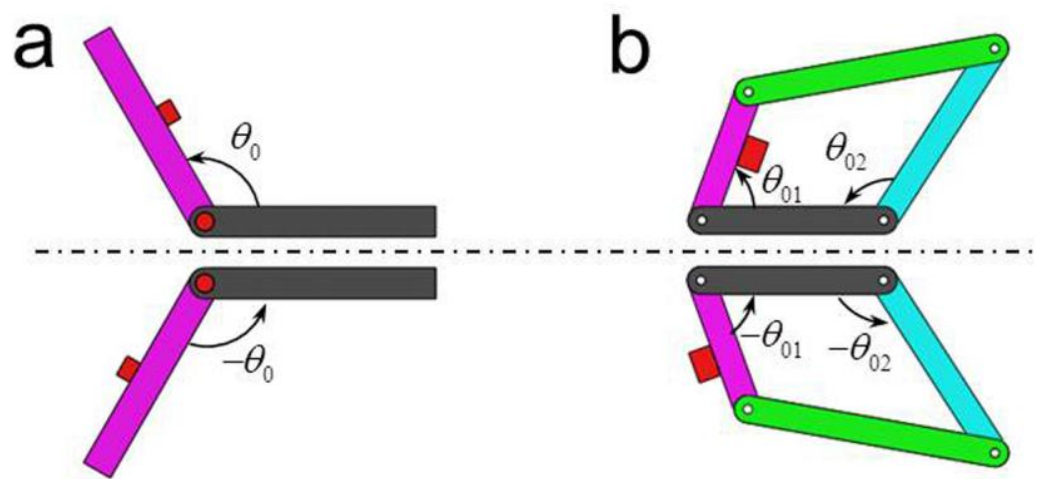

Fig. 4. Explanation of symmetrical (mirrored) configurations distinguished by a designed feature (red box on the purple link). (a) Positive and negative revolute joint angles, and (b) symmetrical configurations of planer four-bar linkage.

In the following section, all of the solutions with negative rotation angles or mirrored configurations are still presented in the figures of solutions even though they look the same as the target configuration. In general, asymmetric design features can confirm the difference easily.

\subsection{Prismatic joint}
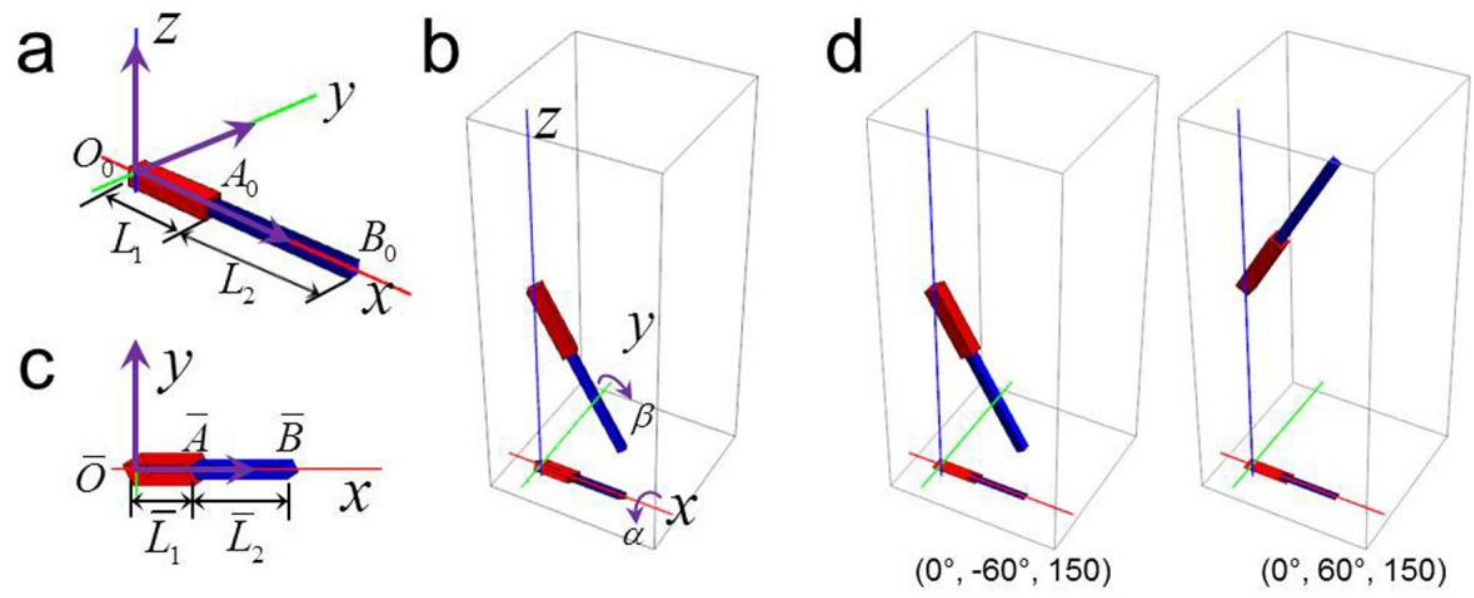

Fig. 5. Projection kinematic analysis of prismatic joint and a numerical example. (a) Coordinate frame of prismatic joint, (b) projection of prismatic joint, (c) projected configuration of the prismatic joint, and (d) solutions of a numerical example of projection kinematics analysis of the prismatic joint. 
Prismatic joints are usually constructed by a slider that translates linearly relative to another part. A simple example is a square rod that slides with a single DOF inside a square tube. Fig. 5a shows the coordinate frame of a general prismatic joint whose cross section is rectangular and the red and blue components are the fixed part and the slider, respectively. The $x$-axis points along the translation direction of the blue slider. From the model, the rotation about $x$-axis does not change projection result if geometrical information of the joint is ignored. $L_{1}$ is the length of the fixed component (red) and $L_{2}$ is the extension of the slider beyond the fixed component, as shown in Fig. 5a. Sketch of the projected configuration of this prismatic joint is shown in Fig. 5 b. In addition, the projection variables, $\bar{L}_{1}$ and $\bar{L}_{2}$ corresponding with $L_{1}$ and $L_{2}$ respectively, are shown in Fig. 5c. By following the same solution procedures applied to the revolute joint, we can obtain two projection kinematics equations for a prismatic joint

$$
\left\{\begin{array}{l}
L_{1} \cos \beta=\bar{L}_{1} \\
\left(L_{1}+L_{2}\right) \cos \beta=\bar{L}_{1}+\bar{L}_{2}
\end{array}\right.
$$

The variable $\alpha$ doesn't show up in Eq. (5) because the rotation about $x$-axis does not affect the projected lengths, $\bar{L}_{1}$ and $\bar{L}_{2}$. Solving Eq. (5) yields two solutions with identical $L_{2}$ but opposite signs of $\beta$ in terms of $L_{1}, \bar{L}_{1}$ and $\bar{L}_{2}$, which means the prismatic joint has a unique configuration with two opposite rotation positions about the $y$-axis that correspond to the same projected configuration. A numerical example for the projection kinematic analysis of prismatic joint is shown in Table 3 and the two solutions are shown in Fig. 5d.

Table 3 Numerical example for projection kinematic analysis of a prismatic joint

\begin{tabular}{|r|r|}
\hline Initial configuration & $L_{1}=100, L_{2}=150$ \\
\hline Rotation angles & $\alpha=-30^{\circ}, \beta=60^{\circ}$ \\
\hline Projected configuration & $\bar{L}_{1}=50, \bar{L}_{2}=75$ \\
\hline
\end{tabular}

\subsection{Cylindrical joint}

The cylindrical joint not only has a translational DOF but also a rotational DOF about the sliding axis. That rotation does not affect the projected configuration if the $x$-axis of global coordinate frame is placed along the rotation axis. In other words, it is impossible to obtain the rotation angle of the cylindrical joint from projected image if geometrical information of the joint is neglected or undistinguishable. Then the projection kinematics analysis is the same as the prismatic joint if its local rotational DOF is ignored.

\subsection{Spherical joint}

A spherical joint as shown in Fig. 6 has three DOFs. Instead of using traditional azimuth and zenith angles for the modeling, here we use angle $\theta_{01}$ between links $O_{0} A_{0}$ and $O_{0} B_{0}$, and angle $\phi_{0}$ of link $O_{0} B_{0}$ rotating about $x$-axis to describe the configuration of spherical joint. In addition, the third DOF, rotation around link $O_{0} B_{0}$, is neglected as it does not affect the projected configuration. Actually, full geometry of the spherical joint is determined only by the angle $\theta_{01}$ if detailed geometries of the two links is negligible. 

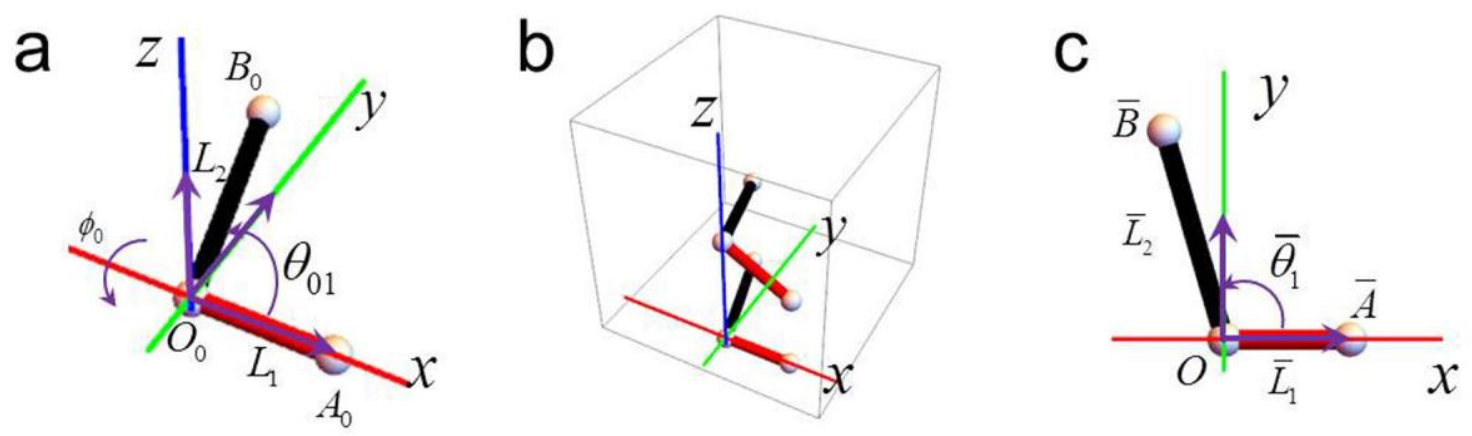

Fig. 6. Projection kinematics analysis of spherical joint. (a) Coordinate frame, (b) projectionfrom z-axis, and (c) the projected configuration .

When the angle $\theta_{01}$ and $\phi_{0}$ are given, the general configuration of the entire spherical joint in space (Fig. 6a) can be determined.

Table 4 Initial, rotated, projected and measured points that define the configuration of spherical joint

\begin{tabular}{|l|l|l|l|}
\hline Points in the local reference frame & Points after rotation & $\begin{array}{l}\text { Projected } \\
\text { points }\end{array}$ & $\begin{array}{l}\text { Measured points from a } \\
\text { projected 2D image }\end{array}$ \\
\hline $\mathbf{A}_{0}=\left(L_{1}, 0,0\right)^{T}$ & $\mathbf{A}=[R] \mathbf{A}_{0}$ & $\overline{\mathbf{A}}=[P] \mathbf{A}$ & $\overline{\mathbf{A}}=\left(\bar{L}_{1}, 0,0\right)^{T}$ \\
\hline $\mathbf{B}_{0}=\left[X\left(\phi_{0}\right)\right]\left(L_{2} \cos \theta_{01}, L_{2} \sin \theta_{01}, 0\right)^{T}$ & $\begin{array}{l}\mathbf{B}=[R] \mathbf{B}_{0} \\
=[Y(\beta)]\left[X\left(\alpha+\phi_{0}\right)\right] \mathbf{B}_{00}\end{array}$ & $\overline{\mathbf{B}}=[P] \mathbf{B}$ & $\overline{\mathbf{B}}=\left(\bar{L}_{2} \cos \bar{\theta}_{1}, \bar{L}_{2} \sin \bar{\theta}_{1}, 0\right)^{T}$ \\
\hline
\end{tabular}

Setting the coordinates of projected points ( $3^{\text {rd }}$ column) equal to the measured points $\left(4^{\text {th }}\right.$ column $)$ in Table 4 , we obtain three projection kinematics equations

$$
\left\{\begin{array}{l}
L_{1} \cos \beta=\bar{L}_{1} \\
L_{2}\left(\cos \theta_{01} \cos \beta+\sin \theta_{01} \cos \left(\alpha+\phi_{0}\right) \sin \beta\right)=\bar{L}_{2} \cos \bar{\theta}_{1} \\
L_{2} \sin \theta_{01} \sin \left(\alpha+\phi_{0}\right)=\bar{L}_{2} \sin \bar{\theta}_{1}
\end{array}\right.
$$

However the above three equations are not sufficient to solve the four unknowns, $\alpha, \beta, \theta_{01}$ and $\phi_{0}$.

Noticing that $\alpha+\phi_{0}$ in Eq. (6) can be treated as a single unknown, eight solutions of $\beta, \theta_{01}$ and $\alpha+\phi_{0}$ can be obtained similarly to the projection kinematics analysis of revolute joint. This agrees with our intuition that the initial configuration is determined by the angle $\theta_{01}$ and local rotation $\phi_{0}$ is coupled to the global rotation about $x$-axis. In other words, the projection kinematics of spherical joint degraded into the same analysis as the revolute joint if specific geometries of the links are neglected.

From the projection kinematic analysis of revolute, prismatic, cylindrical and spherical joints, if the rotation direction is identifiable, for example by an asymmetric design feature, there would be only two configurations with two spatial positions for each joint that can have the same projected configuration for a revolute or a spherical joint. If the rotation of the prismatic about the sliding axis cannot be identified, there will be only one configuration solution with two spatial positions that can have the same projected configuration.

\section{Projection kinematics of planar mechanisms}

Building on the projection kinematics analysis for basic kinematic joints, we can apply the projection kinematics analysis to any planar or spatial mechanisms. Different from the single joint analysis, we consider closed-chain mechanisms with four or more links, which provides more information for analysis of projected configurations. In addition, the DOF of mechanisms can introduce additional constraints to 
the analysis. We first consider a general four-bar linkage and a general five-bar linkage as examples to show the comprehensive analysis procedure.

\subsection{Planar four-bar linkage}

Planar four-bar linkages are constructed by four links connected by four revolute joints. Fig.7a shows the sketch and coordinate frame of a general four bar linkage. As explained previously, thick lines and circles are used to represent the links and joints respectively. We assume the thickness of lines and the size of dots are infinitely small. In reality, geometries of the links or details of the joint may provide constraints to the joint motion. However, this would only simplify the projection kinematics analysis by allowing selection of correct solution. The configuration in space can still be obtained by consequently rotations about $x$-axis and $y$-axis and the rotation matrix is the same as Eq. (2) introduced in section 2.
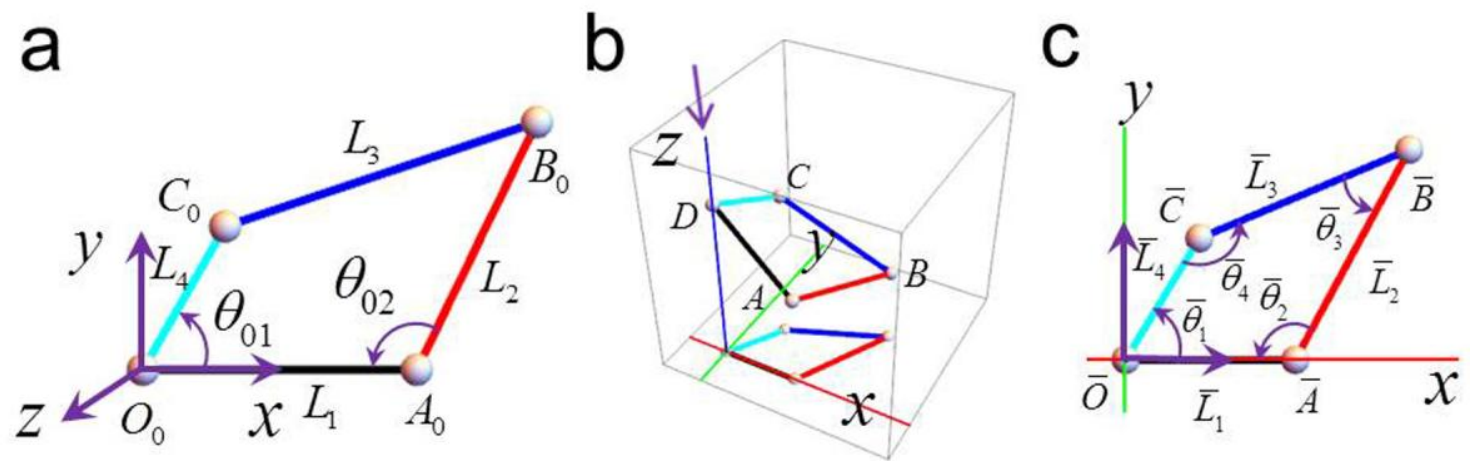

Fig.7. Projection kinematic analysis of a planar four-bar linkage. (a) Local coordinate frame of planar four-bar linkage, (b) original and projected planar four-bar linkage, (c) projected configuration.

In contrast to a single joint, the four-bar linkage has its own kinematics equations which should be solved first to obtain the true configuration if any of the input angles, e.g. $\theta_{01}$, is given. The spatial configuration can be obtained by two global rotation angles $\alpha$ and $\beta$ (Fig. 7b). Hence there are three unknowns in total which means at least three variables must be measured from the projected configuration. Equating the coordinates of projected points $\left(3^{\text {rd }}\right.$ column $)$ and measured points $\left(4^{\text {th }}\right.$ columns $)$ in Table 5 yields five projection kinematic equations,

$$
\begin{aligned}
& L_{1} \cos \beta=\bar{L}_{1} \\
& L_{1} \cos \beta-L_{2} \cos \beta \cos \theta_{02}+L_{2} \sin \alpha \sin \beta \sin \theta_{02}=\bar{L}_{1}+\bar{L}_{2} \cos \left(\pi-\bar{\theta}_{2}\right) \\
& L_{2} \cos \alpha \sin \theta_{02}=\bar{L}_{2} \sin \left(\pi-\bar{\theta}_{2}\right) \\
& L_{4} \cos \beta \cos \theta_{01}+L_{4} \sin \alpha \sin \beta \sin \theta_{01}=\bar{L}_{4} \cos \bar{\theta}_{1} \\
& L_{4} \cos \alpha \sin \theta_{01}=\bar{L}_{4} \sin \bar{\theta}_{1}
\end{aligned}
$$

And the geometric constraint equation of the constant coupler link length is $\left|\mathbf{B}_{0}-\mathbf{C}_{0}\right|-L_{3}=0$, which can be rewritten as the following equation after substituting the coordinates of $\mathbf{B}_{0}$ and $\mathbf{C}_{0}$,

$$
L_{1}^{2}+L_{2}^{2}+L_{4}^{2}-L_{3}^{2}-2 L_{1} L_{4} \cos \theta_{01}-2 L_{1} L_{2} \cos \theta_{02}+2 L_{2} L_{4} \cos \left(\theta_{01}+\theta_{02}\right)=0
$$

Including the geometry constraint equation Eq.(12), six equations are obtained and $\theta_{01}, \theta_{02}, \alpha$, and $\beta$ are the unknowns while $\bar{\theta}_{1}, \bar{\theta}_{2}, \bar{L}_{1}, \bar{L}_{2}$ and $\bar{L}_{4}$ can be measured from the projected configuration. 
Table 5 Initial, rotated, projected and measured points that define the configuration of planar four-bar linkage

\begin{tabular}{|l|l|l|l|}
\hline $\begin{array}{l}\text { Points in the local reference } \\
\text { frame }\end{array}$ & $\begin{array}{l}\text { Points after } \\
\text { rotation }\end{array}$ & Projected points & $\begin{array}{l}\text { Measured points from a 2D } \\
\text { image }\end{array}$ \\
\hline $\mathbf{A}_{0}=\left(L_{1}, 0,0\right)^{T}$ & $\mathbf{A}=[R] \mathbf{A}_{0}$ & $\overline{\mathbf{A}}=[P] \mathbf{A}$ & $\overline{\mathbf{A}}=\left(\bar{L}_{1}, 0,0\right)^{T}$ \\
\hline $\begin{array}{l}\mathbf{B}_{0}=\mathbf{A}_{0}+ \\
L_{2}\left(\cos \left(\pi-\theta_{02}\right), \sin \left(\pi-\theta_{02}\right), 0\right)^{T}\end{array}$ & $\mathbf{B}=[R] \mathbf{B}_{0}$ & $\overline{\mathbf{B}}=[P] \mathbf{B}$ & $\begin{array}{l}\overline{\mathbf{B}}=\overline{\mathbf{A}}+ \\
\left(\bar{L}_{2} \cos \left(\pi-\bar{\theta}_{2}\right), \bar{L}_{2} \sin \left(\pi-\bar{\theta}_{2}\right), 0\right)^{T}\end{array}$ \\
\hline $\mathbf{C}_{0}=L_{4}\left(\cos \theta_{01}, \sin \theta_{01}, 0\right)^{T}$ & $\mathbf{C}=[R] \mathbf{C}_{0}$ & $\overline{\mathbf{C}}=[P] \mathbf{C}$ & $\overline{\mathbf{C}}=\left(\bar{L}_{4} \cos \bar{\theta}_{1}, \bar{L}_{4} \sin \bar{\theta}_{1}, 0\right)^{T}$ \\
\hline
\end{tabular}

Notice that there are eight total parameters including four angles, $\bar{\theta}_{1}, \bar{\theta}_{2}, \bar{\theta}_{3}$ and $\bar{\theta}_{4}$, and four link lengths, $\bar{L}_{1}, \bar{L}_{2}, \bar{L}_{3}$ and $\bar{L}_{4}$, which can be measured on the projected configuration (Fig. 8c). This gives a total of 56 combinations of three parameters that can be chosen from the total of eight. However, we only need to consider the following four cases without loss of generality.

1. Two adjacent links and the joint between them. For example, if $\bar{L}_{1}, \bar{L}_{4}$ and $\bar{\theta}_{1}$ are measured, then Eqs. $(7,10,11)$ and constraint equation Eq. (12) are enough to obtain $\theta_{01}, \theta_{02}, \alpha, \beta$. The solution process is identical to that of the revolute joint.

2. Two adjacent joint angles and the length of link between them. For instance, if $\bar{\theta}_{1}, \bar{L}_{1}$ and $\bar{\theta}_{2}$ are measured, $\bar{L}_{2}$ can be obtained first by Eq. (9)

$$
\bar{L}_{2}=L_{2} \cos \alpha \sin \theta_{02} / \sin \left(\pi-\bar{\theta}_{2}\right)
$$

Substituting (13) into (8) yields

$$
L_{1} \cos \beta-L_{2} \cos \beta \cos \theta_{02}+L_{2} \sin \alpha \sin \beta \sin \theta_{02}=\bar{L}_{1}+\left(L_{2} \cos \alpha \sin \theta_{02} / \sin \left(\pi-\bar{\theta}_{2}\right)\right) \cos \left(\pi-\bar{\theta}_{2}\right)
$$

and $\bar{L}_{4}$ can be obtained from Eq. (11)

$$
\bar{L}_{4}=L_{4} \cos \alpha \sin \theta_{01} / \sin \bar{\theta}_{1}
$$

Substituting (15) into (10) yields

$$
L_{4} \cos \beta \cos \theta_{01}+L_{4} \sin \alpha \sin \beta \sin \theta_{01}=\left(L_{4} \cos \alpha \sin \theta_{01} / \sin \bar{\theta}_{1}\right) \cos \bar{\theta}_{1}
$$

Finally, Eqs. $(7,14,16)$ and constraint equation Eq. (12) are enough to obtain $\theta_{01}, \theta_{02}, \alpha, \beta$.

3. Three joint angles are measured, e.g. $\bar{\theta}_{1}, \bar{\theta}_{2}, \bar{\theta}_{3}$. In addition, the projected configuration has another close loop kinematic constraint, written as

$$
\begin{aligned}
\bar{L}_{1}+\bar{L}_{2} \cos \left(\pi-\bar{\theta}_{2}\right) & =\bar{L}_{4} \cos \bar{\theta}_{1}+\bar{L}_{3} \cos \left(\psi_{3}\right) \\
\bar{L}_{2} \sin \left(\pi-\bar{\theta}_{2}\right) & =\bar{L}_{4} \sin \bar{\theta}_{1}+\bar{L}_{3} \sin \left(\psi_{3}\right)
\end{aligned}
$$

where

$$
\begin{aligned}
& \bar{\theta}_{4}=2 \pi-\left(\bar{\theta}_{1}+\bar{\theta}_{2}+\bar{\theta}_{3}\right) \\
& \psi_{3}=\bar{\theta}_{1}+\bar{\theta}_{4}-\pi
\end{aligned}
$$


and $\bar{L}_{3}$ can be obtained by substituting $\bar{L}_{2}$ in Eq. (13) and $\bar{L}_{4}$ in Eq. (15) into Eq. (18)

$$
\bar{L}_{3}=\left(L_{2} \cos \alpha \sin \theta_{02}-L_{4} \cos \alpha \sin \theta_{01}\right) / \sin \left(\psi_{3}\right)
$$

Then $\bar{L}_{1}, \bar{L}_{2}, \bar{L}_{4}$ and $\bar{L}_{3}$ were obtained from Eqs. $(7,13,15,20)$ and substituted into Eqs. $(8,10,17)$ to obtain

$L_{1} \cos \beta-L_{2} \cos \beta \cos \theta_{02}+L_{2} \sin \alpha \sin \beta \sin \theta_{02}=\left(L_{1} \cos \beta\right)+\left(L_{2} \cos \alpha \sin \theta_{02} / \sin \left(\pi-\bar{\theta}_{2}\right)\right) \cos \left(\pi-\bar{\theta}_{2}\right)$

$$
L_{4} \cos \beta \cos \theta_{01}+L_{4} \sin \alpha \sin \beta \sin \theta_{01}=\left(L_{4} \cos \alpha \sin \theta_{01} / \sin \bar{\theta}_{1}\right) \cos \bar{\theta}_{1}
$$

$$
L_{1} \cos \beta+L_{2} \cos \alpha \sin \theta_{02} \cot \left(\pi-\bar{\theta}_{2}\right)=L_{4} \cos \alpha \sin \theta_{01} \cot \bar{\theta}_{1}+\left(L_{2} \cos \alpha \sin \theta_{02}-L_{4} \cos \alpha \sin \theta_{01}\right) \cot \left(\psi_{3}\right)
$$

Eqs $(21,22,23)$ and Eq. (12) are the final four equations that are used to obtain $\theta_{01}, \theta_{02}, \alpha, \beta$.

4. Three adjacent links, e.g. $\bar{L}_{1}, \bar{L}_{2}$ and $\bar{L}_{4}$ are measured. Based on two projected link lengths, i.e. $|\overline{\mathbf{O}}-\overline{\mathbf{C}}|=\bar{L}_{4},|\overline{\mathbf{A}}-\overline{\mathbf{B}}|=\bar{L}_{2}$, we can obtain

$$
\begin{aligned}
& \left(L_{4} \cos \beta \cos \theta_{01}+L_{4} \sin \alpha \sin \beta \sin \theta_{01}\right)^{2}+\left(L_{4} \cos \alpha \sin \theta_{01}\right)^{2}=\bar{L}_{4}^{2} \\
& \left(-L_{2} \cos \beta \cos \theta_{02}+L_{2} \sin \alpha \sin \beta \sin \theta_{02}\right)^{2}+\left(L_{2} \cos \alpha \sin \theta_{02}\right)^{2}=\bar{L}_{2}^{2}
\end{aligned}
$$

Together with Eq. $(7,12)$, we can solve for the four unknowns, $\theta_{01}, \theta_{02}, \alpha, \beta$.

The four cases for projection kinematic analysis of four-bar linkage and the corresponding equations are summarized in Table 6. Note each case is a representative case of multiple permutations. As shown in Table 6, the number of solutions determines the complexity of the set equations to be solved. And the last column represents the actual solutions that satisfy all measured geometrical information.

Table 6 Strategies for projection kinematic analysis of four-bar linkage

\begin{tabular}{|c|l|l|l|l|l|}
\hline Case & $\begin{array}{l}\text { Measured geometric } \\
\text { parameters }\end{array}$ & $\begin{array}{l}\text { Representative } \\
\text { measured parameters }\end{array}$ & $\begin{array}{l}\text { Representative } \\
\text { equations }\end{array}$ & $\begin{array}{l}\text { \# of } \\
\text { solutions }\end{array}$ & $\begin{array}{l}\text { \# of good } \\
\text { solutions }\end{array}$ \\
\hline 1 & $\begin{array}{l}\text { Two links and the joint } \\
\text { angle between them }\end{array}$ & $\bar{L}_{1}, \bar{L}_{4}, \bar{\theta}_{1}$ & $(7,10,11,12)$ & 8 & 2 \\
\hline 2 & $\begin{array}{l}\text { Two adjacent joint angles } \\
\text { and the link between them }\end{array}$ & $\bar{\theta}_{1}, \bar{L}_{1}, \bar{\theta}_{2}$ & $(7,12,15,16)$ & 16 & 4 \\
\hline 3 & Three joint angles & $\bar{\theta}_{1}, \bar{\theta}_{2}, \bar{\theta}_{3}$ & $(12,21,22,23)$ & 96 & 4 \\
\hline 4 & Three links & $\bar{L}_{1}, \bar{L}_{2}, \bar{L}_{4}$ & $(7,12,24,25)$ & 96 & 8 \\
\hline
\end{tabular}

A numerical example is discussed below to verify the strategies introduced above. The variables of initial and projected configurations and global rotations are shown in Table 7.

Table 7 Numerical example of projection kinematic analysis of a four-bar linkage

\begin{tabular}{|l|l|}
\hline Initial configuration & $L_{1}=5, L_{2}=5, L_{3}=6, L_{4}=3, \theta_{01}=60^{\circ}, \theta_{02}=116.011^{\circ} ; \alpha=15^{\circ}, \beta=45^{\circ}$ \\
\hline Projected configuration & $\begin{array}{l}\bar{L}_{1}=3.54, \bar{L}_{2}=4.95, \bar{L}_{3}=4.74, \bar{L}_{4}=2.94 ; \bar{\theta}_{1}=58.53^{\circ}, \bar{\theta}_{2}=118.67^{\circ}, \bar{\theta}_{3} \\
=38.61^{\circ} ;\end{array}$ \\
\hline
\end{tabular}


The equations in cases 1 and 2 can be solved easily. However, the equations derived in cases 3 and 4 are too complex to be solved directly. Instead, we used the polynomial homotopy solver PHCpack [24] to find the solutions of cases 3 and 4 . To obtain polynomial equations, we defined $c$ and $s$ for $\cos (\bullet)$ and $\sin (\bullet)$ functions and introduce the extra identical equation $c^{2}+s^{2}=1$ for each angle variable. Finally, all solutions of cases 1, 2, 3 and 4 are summarized in Appendix material Table A1. For each solution, the spatial and projected configurations are presented in a boxed figure and the values of the unknowns, $\left(\theta_{01}\right.$, $\left.\theta_{02}, \alpha, \beta\right)$ are shown at the bottom of each boxed figure.

\subsection{Planar five-bar linkage}

In order to further test the projection kinematics routine, we considered a general planar five-bar linkage. Here we focus on only one case, i.e. measuring joint angles only. The planar five-bar linkage has two DOFs that will bring in two extra input unknowns and together with the two global rotation variables, four total unknowns need to be solved, which means we need to measure at least four angles to solve the projection kinematics equations.
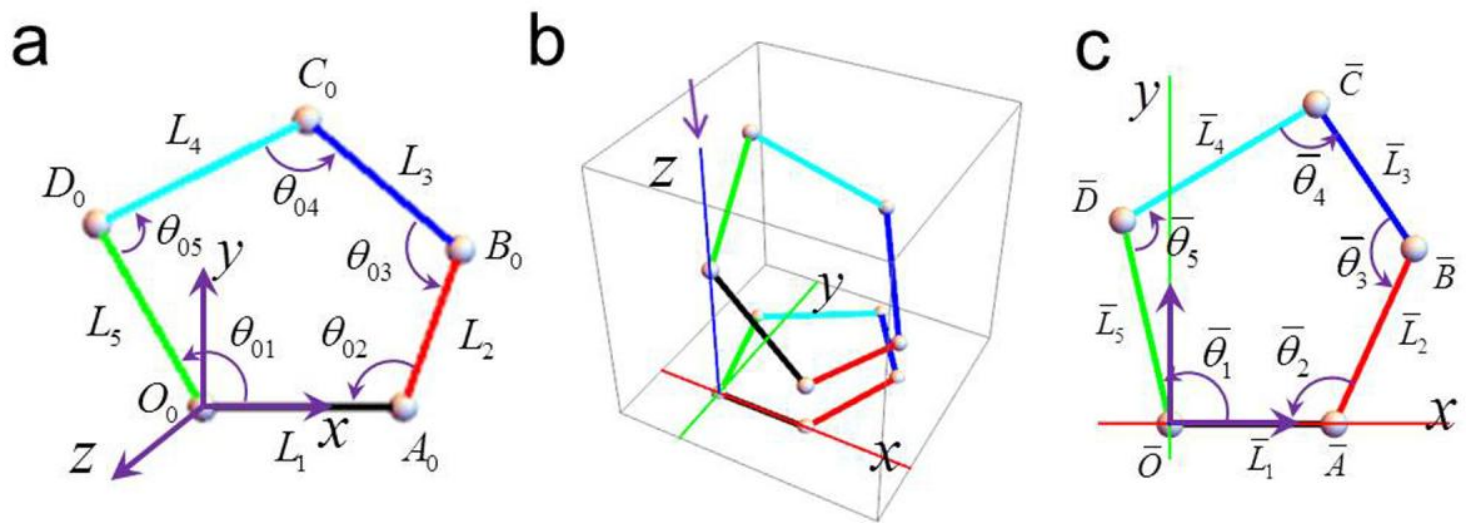

Fig. 8. Projection kinematics analysis of a planar five-bar linkage. (a) Local coordinate frame is defined, (b) planar five-bar linkage in space and its projection onto $x y$ plane, and (c) projected configuration.

A planar five-bar linkage consists of five links connected by five revolute joints (Fig. 8a), when $\theta_{01}$ and $\theta_{02}$ are given, the configuration of the five-bar linkage is determined but not unique due to the existence of multiple solutions of $\theta_{03}$. To uniquely determine the true configuration, we must rely on extra information to pick the correct solution of $\theta_{03}$. Fig. $8 \mathrm{~b}$ shows the sketch of the projection after global rotations about $x$ axis and $y$-axis and detailed projected configuration is shown in Fig. 8c. Four adjacent joint angles, $\bar{\theta}_{1}, \bar{\theta}_{2}$, $\bar{\theta}_{3}$ and $\bar{\theta}_{4}$ were measured and used for the analysis. Table 8 presents the joints positions of initial, rotated and projected configurations of the five-bar linkage.

Table 8 Initial, rotated, projected and measured points that define the configuration of planar five-bar linkage

\begin{tabular}{|l|l|l|l|}
\hline Points in the local reference frame & $\begin{array}{l}\text { Points after } \\
\text { rotation }\end{array}$ & $\begin{array}{l}\text { Projected } \\
\text { points }\end{array}$ & Measured points from a 2D image \\
\hline $\mathbf{A}_{0}=\left(L_{1}, 0,0\right)^{T}$ & $\mathbf{A}=[R] \mathbf{A}_{0}$ & $\overline{\mathbf{A}}=[P] \mathbf{A}$ & $\overline{\mathbf{A}}=\left(\bar{L}_{1}, 0,0\right)^{T}$ \\
\hline $\begin{array}{l}\mathbf{B}_{0}=\mathbf{A}_{0}+ \\
L_{2}\left(\cos \left(\pi-\theta_{02}\right), \sin \left(\pi-\theta_{02}\right), 0\right)^{T}\end{array}$ & $\mathbf{B}=[R] \mathbf{B}_{0}$ & $\overline{\mathbf{B}}=[P] \mathbf{B}$ & $\begin{array}{l}\overline{\mathbf{B}}=\overline{\mathbf{A}}+ \\
\left(\bar{L}_{2} \cos \left(\pi-\bar{\theta}_{2}\right), \bar{L}_{2} \sin \left(\pi-\bar{\theta}_{2}\right), 0\right)^{T}\end{array}$ \\
\hline $\begin{array}{l}\mathbf{C}_{0}=\mathbf{B}_{0}+ \\
\left(L_{3} \cos \left(\theta_{02}+\theta_{03}\right),-L_{3} \sin \left(\theta_{02}+\theta_{03}\right), 0\right)^{T}\end{array}$ & $\mathbf{C}=[R] \mathbf{C}_{0}$ & $\overline{\mathbf{C}}=[P] \mathbf{C}$ & $\begin{array}{l}\overline{\mathbf{C}}=\overline{\mathbf{B}}+ \\
\left(\bar{L}_{3} \cos \left(\bar{\theta}_{2}+\bar{\theta}_{3}\right),-\bar{L}_{3} \sin \left(\bar{\theta}_{2}+\bar{\theta}_{3}\right), 0\right)^{T}\end{array}$ \\
\hline
\end{tabular}




$$
\begin{array}{|l|l|l|l}
\hline \mathbf{D}_{0}=\left(L_{5} \cos \theta_{01}, L_{5} \sin \theta_{01}, 0\right)^{T} & \mathbf{D}=[R] \mathbf{D}_{0} & \overline{\mathbf{D}}=[P] \mathbf{D} & \overline{\mathbf{D}}=\left(\bar{L}_{5} \cos \bar{\theta}_{1}, \bar{L}_{5} \sin \bar{\theta}_{1}, 0\right)^{T}
\end{array}
$$

Equating the coordinates of projected points ( $3^{\text {rd }}$ column) to measured points ( $4^{\text {th }}$ column $)$ in Table 8 yields seven equations,

$$
\left\{\begin{array}{l}
L_{1} \cos \beta=\bar{L}_{1} \\
L_{1} \cos \beta-L_{2} \cos \beta \cos \theta_{02}+L_{2} \sin \alpha \sin \beta \sin \theta_{02}=\bar{L}_{1}+\bar{L}_{2} \cos \left(\pi-\bar{\theta}_{2}\right) \\
L_{2} \cos \alpha \sin \theta_{02}=\bar{L}_{2} \sin \left(\pi-\bar{\theta}_{2}\right) \\
L_{1} \cos \beta-L_{2} \cos \beta \cos \theta_{02}+L_{3} \cos \beta \cos \left(\theta_{02}+\theta_{03}\right)+L_{2} \sin \alpha \sin \beta \sin \theta_{02}-L_{3} \sin \alpha \sin \beta \sin \left(\theta_{02}+\theta_{03}\right) \\
=\bar{L}_{1}+\bar{L}_{2} \cos \left(\pi-\bar{\theta}_{2}\right)+\bar{L}_{3} \cos \left(\bar{\theta}_{2}+\bar{\theta}_{3}\right), \\
L_{2} \cos \alpha \sin \theta_{02}-L_{3} \cos \alpha \sin \left(\theta_{02}+\theta_{03}\right)=\bar{L}_{2} \sin \left(\pi-\bar{\theta}_{2}\right)-\bar{L}_{3} \sin \left(\bar{\theta}_{2}+\bar{\theta}_{3}\right) \\
L_{5} \cos \beta \cos \theta_{01}+L_{5} \sin \alpha \sin \beta \sin \theta_{01}=\bar{L}_{5} \cos \bar{\theta}_{1} \\
L_{5} \cos \alpha \sin \theta_{01}=\bar{L}_{5} \sin \bar{\theta}_{1}
\end{array}\right.
$$

Based on the coordinate frame in Fig. 9a, the geometry constraint equation is

$$
\left|\mathbf{C}_{0}-\mathbf{D}_{0}\right|-L_{4}^{2}=0
$$

In addition, the projected configuration also forms a closed-loop mechanism, which provides two additional constraint equations

$$
\left\{\begin{array}{l}
\bar{L}_{1}-\bar{L}_{2} \cos \bar{\theta}_{2}+\bar{L}_{3} \cos \left(\bar{\theta}_{2}+\bar{\theta}_{3}\right)-\bar{L}_{5} \cos \bar{\theta}_{1}+\bar{L}_{4} \cos \left(\bar{\theta}_{1}+\bar{\theta}_{5}\right)=0 \\
\bar{L}_{2} \sin \bar{\theta}_{2}-\bar{L}_{3} \sin \left(\bar{\theta}_{2}+\bar{\theta}_{3}\right)-\bar{L}_{5} \sin \bar{\theta}_{1}+\bar{L}_{4} \sin \left(\bar{\theta}_{1}+\bar{\theta}_{5}\right)=0
\end{array}\right.
$$

Finally, we obtained ten total equations, Eqs. $(26,27,28)$, for the projection kinematics analysis of the planar five-bar mechanism. First, $\bar{L}_{1}, \bar{L}_{2}, \bar{L}_{3}, \bar{L}_{4}$, and $\bar{L}_{5}$ should be eliminated and only five equations with five unknowns, $\theta_{01}, \theta_{02}, \theta_{03}, \alpha$ and $\beta$, are left. Then the five equations, along with the five identical equation $c^{2}+s^{2}=1$ for each angle variable can be solved by the PHCpack solver. The redundant solutions were eliminated by checking the four measured projected joint angles and the projected link lengths. A numerical example of projection analysis of a five-bar linkage was shown in Appendix Table A2. Eight solutions were obtained and shown in Appendix Fig. A1. The first four solutions have the same configurations as designed but with four different orientations in 3D space, while the last four solutions are the mirrored configurations, also with four different orientations.

\section{Algorithm of the projection kinematics analysis}

All of the projection kinematics equations can be solved when the number of unknowns equals to the number of equations, which is referred as the minimum measurement case. With minimum measurements, the projection kinematics analysis procedure is summarized as follows:

a) Set up the global coordinate frame of the mechanism. Specifically, it is convenient to place the $x$ axis along a link so that the projected link length of this link will not change if the entire mechanism has a rotation about $x$-axis.

b) Define the true configuration of the mechanism.

c) Conduct the global rotation about $x$-axis and $y$-axis to obtain the spatial configuration.

d) Project the spatial configuration to the $x y$ plane and obtain the symbolic equations of the projected angles and projected link lengths. 
e) Measure the corresponding angles and link lengths from the experimental 2D image, e.g. a TEM image.

f) Obtain the minimum projection kinematics equations by comparing the variables obtained in d) and e).

g) Solve the projection kinematics equations obtained in f). PHCpack solver can be used when the equations are complex.

h) Compare the projection information derived from the solutions obtained in $g$ ) with the measured ones obtained in e) and keep the correct solutions if the differences between them are acceptable.

In real world applications, you may have more than minimum measurements. Different measurements for the projection kinematics equations may result in different solutions with different errors. Errors mainly depend on the fabrication of the mechanisms and accuracy of measurement. In order to minimize the errors, the variables with less measurement errors should been used firstly and the quality of the solutions must be verified by extra kinematics equations or measurements. For example, $\left\{Q_{m}=0\right\}$ and $\left\{\bar{x}_{n}\right\}$ are respectively the collection of all projection kinematics equations and measurements, and $\left\{Q_{j}=0\right\}$ is a subset of $\left\{Q_{m}=0\right\}$ and has the minimum number of equations corresponding to the chosen subset of measurements $\left\{\bar{x}_{j}\right\}$. The error can be quantified by substituting solutions obtained by solving $\left\{Q_{j}=0\right\}$ into all other kinematics equations, as $\varepsilon=\sum Q_{k}(k \neq j)$. Also, the error can be termed by the derived projection variables and the extra measurements as $\delta=\sum\left(\bar{x}_{i}-\bar{x}_{i-P}\right) / \bar{x}_{i}(i \neq j)$. Finally, the good solutions should be those that can obtain small and acceptable errors $\varepsilon$ and $\delta$.

\section{Application to configuration estimation of DNA origami nanomechanisms}

In this section, we will apply projection kinematics to the analysis of two real DNA origami mechanisms (DOM). As mentioned in the introduction section, the motivation of this research comes from the need to estimate the true configuration of DOM from a single 2D microscopy image, which is essentially the projected image of an arbitrary mechanism in 3D space. Errors of variables measured from TEM images always exist due to the fuzzy ends or boundaries of double strand DNA bundles. The main sources of the fuzzy end or boundaries include ssDNA at the end of the link (to prevent nonspecific binding) or staining during TEM sample preparation [7,8].

Fig. 9 shows how the projected angle and link length are measured from a TEM image and red lines are the reference lines that parallel with the helices of double strand DNA on each link. It is usually very easy to distinguish the helices of double strand DNA on TEM image, such as the four helices (labeled as 1, 2, 3 and 4) on the upper left link. The angle $\theta$ between the reference lines is measured as the projected angle and the projection link length is measured along the reference line. However, the ends of double strand DNA bundles are fuzzy. Therefore, estimated positions will be chosen for the length measurement. For example, the two ends of the horizontal link on Fig. 9 fall in the two white boxes and the width of the white box is about $12 \mathrm{~nm}$ which is close to $20 \%$ of the designed link length. Here, the projection kinematics results are acceptable if the link length error between the calculation and the measurement is smaller or close to $20 \%$. 


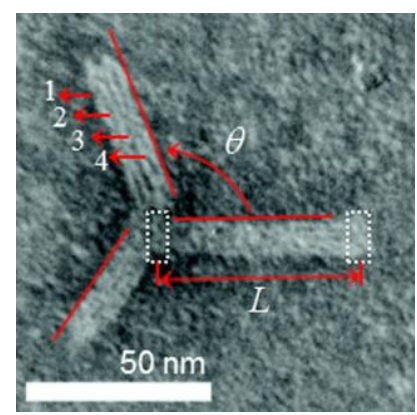

Fig. 9. Measurements on TEM image.

\subsection{The DNA origami universal joint TEM image analysis}
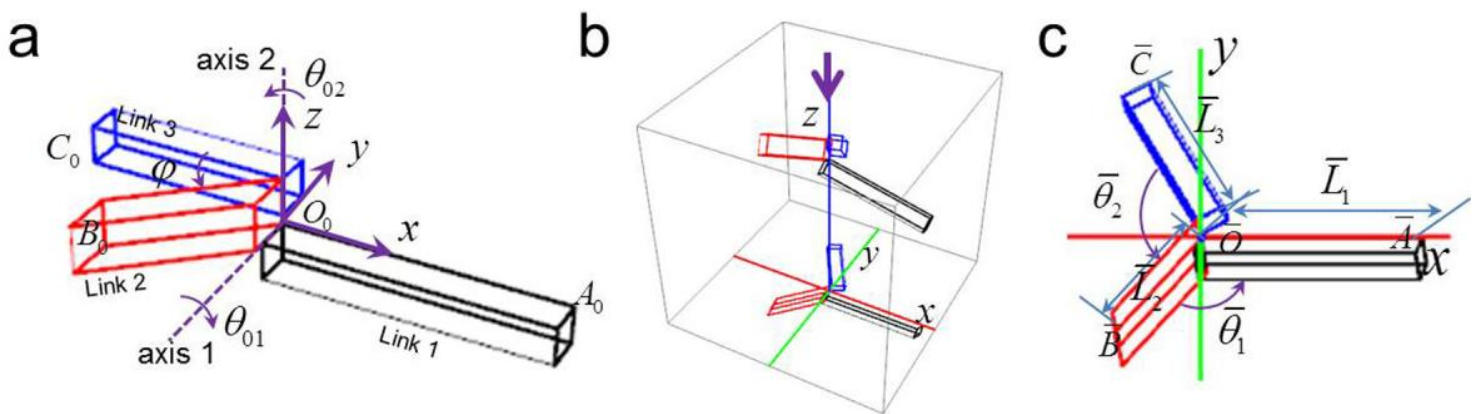

Fig. 10. Projection kinematics analysis of DNA origami universal joint. (a, b, c) are the coordinate frame, sketch of the projection and projected configuration of the DNA origami universal joint respectively.

The design of a universal joint with DNA origami nanotechnology has been described in [7]. Here, the universal joint was used as an example of an open chain mechanism for demonstrating the application of projection kinematics for the analysis of 2D transmission electron microscopy (TEM) images. The dimension information is also represented in the Appendix material Fig. A2. In addition, the conceptual design of the universal joint and the initial position are shown in Fig. 10a. Axis 1, constructed by the shared edge between link 1 and link 2, and axis 2, constructed by the edge between link 2 and link 3 , intersect at a point, which is also chosen as the origin position of the global coordinate frame. $\theta_{01}$ and $\theta_{02}$ are the initial rotation angles of the two joints respectively. To be clear, the spatial and projected configurations are shown in Fig. 10b and 10c respectively.

Here, $\mathrm{A}_{0}, \mathrm{~B}_{0}$ and $\mathrm{C}_{0}$ were picked on the three links respectively as the primary points to explain the solution process. Other points on each link could also be used following a similar computing procedure. The rotation matrix about the two hinge joints were defined as

$$
\left[Y\left(\theta_{01}\right)\right]=\left[\begin{array}{ccc}
\cos \theta_{01} & 0 & \sin \theta_{01} \\
0 & 1 & 0 \\
-\sin \theta_{01} & 0 & \cos \theta_{01}
\end{array}\right],\left[Z\left(\theta_{02}\right)\right]=\left[\begin{array}{ccc}
\cos \theta_{02} & -\sin \theta_{02} & 0 \\
\sin \theta_{02} & \cos \theta_{02} & 0 \\
0 & 0 & 1
\end{array}\right]
$$

Then the coordinates of points $\mathrm{A}_{0}, \mathrm{~B}_{0}$ and $\mathrm{C}_{0}$ on the initial, rotated and projected configurations are derived and presented in Table 9.

Table 9 Initial, rotated, projected and measured points that define the structure of the DNA origami universal joint

\begin{tabular}{|l|l|l|l|}
\hline Initial configuration points & Points after rotation & Projected & Measured points from a 2D \\
\hline
\end{tabular}




\begin{tabular}{|l|l|l|l|}
\hline & & points & image \\
\hline $\mathbf{A}_{0}=\left(L_{1}, 0,0\right)^{T}$ & $\mathbf{A}=[R] \mathbf{A}_{0}$ & $\overline{\mathbf{A}}=[P] \mathbf{A}$ & $\overline{\mathbf{A}}=\left(\bar{L}_{1}, 0,0\right)^{T}$ \\
\hline $\mathbf{B}_{0}=\left(-L_{2} \cos \varphi,-L_{2} \sin \varphi, 0\right)^{T}$ & $\mathbf{B}=[R]\left[Y\left(\theta_{01}\right)\right] \mathbf{B}_{0}$ & $\overline{\mathbf{B}}=[P] \mathbf{B}$ & $\overline{\mathbf{B}}=\left(\bar{L}_{2} \cos \bar{\theta}_{1},-\bar{L}_{2} \sin \bar{\theta}_{1}, 0\right)^{T}$ \\
\hline $\mathbf{C}_{0}=\left(-L_{3}, 0, \Delta h\right)^{T}$ & $\mathbf{C}=[R]\left[Y\left(\theta_{01}\right)\right]\left[Z\left(\theta_{02}\right)\right] \mathbf{C}_{0}$ & $\overline{\mathbf{C}}=[P] \mathbf{C}$ & $\begin{array}{l}\overline{\mathbf{C}}=\left(\bar{L}_{3} \cos \left(\bar{\theta}_{1}+\bar{\theta}_{2}\right),\right. \\
\left.-\bar{L}_{3} \sin \left(\bar{\theta}_{1}+\bar{\theta}_{2}\right), 0\right)^{T}\end{array}$ \\
\hline
\end{tabular}

Note: $\Delta h$ is a design variable that is shown in Appendix Figure A2.

Equating the coordinates of projected $\left(3^{\text {rd }}\right.$ column) to measured points $\left(4^{\text {th }}\right.$ column $)$ in Table 9 , five equations were obtained,

$$
\left\{\begin{array}{l}
L_{1} \cos \beta=\bar{L}_{1} \\
L_{2}\left(-\cos \beta \cos \theta_{01} \cos \varphi+\cos \alpha \sin \beta \sin \theta_{01} \cos \varphi-\sin \alpha \sin \beta \sin \varphi\right)=\bar{L}_{2} \cos \bar{\theta}_{1} \\
L_{2}\left(\sin \alpha \sin \theta_{01} \cos \varphi+\cos \alpha \sin \varphi\right)=\bar{L}_{2} \sin \bar{\theta}_{1} \\
L_{3}\left(-\cos \beta \cos \theta_{01} \cos \theta_{02}+\cos \alpha \sin \beta \sin \theta_{01} \cos \theta_{02}-\sin \alpha \sin \beta \sin \theta_{02}\right)=\bar{L}_{3} \cos \left(\bar{\theta}_{1}+\bar{\theta}_{2}\right) \\
L_{3}\left(-\sin \alpha \sin \theta_{01} \cos \theta_{02}-\cos \alpha \sin \theta_{02}\right)=-\bar{L}_{3} \sin \left(\bar{\theta}_{1}+\bar{\theta}_{2}\right)
\end{array}\right.
$$

The unknowns $\alpha, \beta, \theta_{01}$ and $\theta_{02}$ can be easily solved from Eq. (30) if $\bar{\theta}_{1}, \bar{\theta}_{2}, \bar{L}_{1}$, and $\bar{L}_{2}$ are measured from the projected configuration. The solution of $\bar{L}_{3}$ can be used as a criterion for picking the correct configuration.
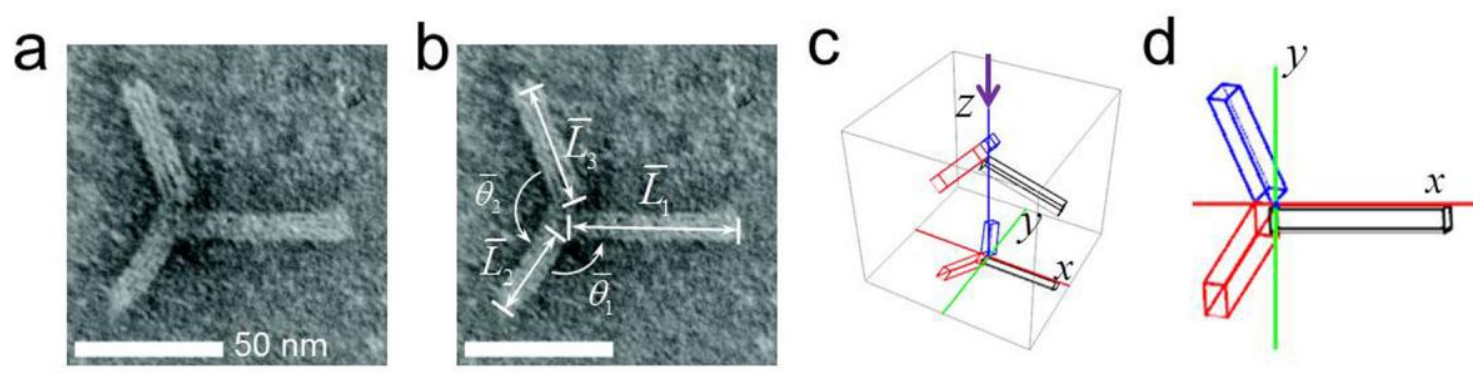

Fig. 11. Projection kinematics analysis of the universal joint based on the TEM image. (a) A typical TEM image of the DNA origami universal joint. (b) Projection information that can be measured from the TEM image. (c) 3D projection solution of the TEM image sample. (d) Detailed projected configuration of the solution.

Here, one typical TEM image of DNA origami universal joint is shown in Fig. 11a. $\bar{\theta}_{1}, \bar{\theta}_{2}, \bar{L}_{1}, \bar{L}_{2}$ and $\bar{L}_{3}$ were measured from the TEM image (Fig. 11b) as shown in Table 10. All measurements were conducted by ImageJ [25] and the value of each measured variable was the average value of three repeated measurements. Finally, sixteen solutions were obtained and only the one that corresponds to the minimum difference between measured $\bar{L}_{3}$ and derived projection link length $\bar{L}_{3-P}$ was kept as the right solution.

Here, the difference between $\bar{L}_{3}$ and $\bar{L}_{3-P}$ is about $4.42 \%$. Fig. 11c shows the 3D projection of the solution and Fig. 11d shows the detailed projected configuration of the DNA origami universal joint.

Table 10 Projection kinematics analysis of a DNA origami universal joint

\begin{tabular}{|l|l|}
\hline True configuration & $L_{1}=59.84 \mathrm{~nm}, L_{2}=34.34 \mathrm{~nm}, L_{3}=49.3 \mathrm{~nm} ; \varphi=41.75^{\circ}$ \\
\hline Projected configuration & $\bar{L}_{1}=57.29, \bar{L}_{2}=31.38, \bar{L}_{3}=37.52, \bar{\theta}_{1}=122.20^{\circ}, \bar{\theta}_{2}=123.49^{\circ}$ \\
\hline Solution & $\theta_{01}=-62.12^{\circ}, \theta_{02}=-62.99^{\circ} ; \alpha=-10.32^{\circ}, \beta=16.79^{\circ}$ \\
\hline
\end{tabular}


Projected variables calculated from the solution
$\bar{L}_{1-P}=57.29, \bar{L}_{2-P}=31.38, \bar{L}_{3-P}=39.18, \bar{\theta}_{1-P}=122.20^{\circ}, \bar{\theta}_{2-P}=$ $123.49^{\circ}$

\subsection{The DNA origami Bennett linkage}
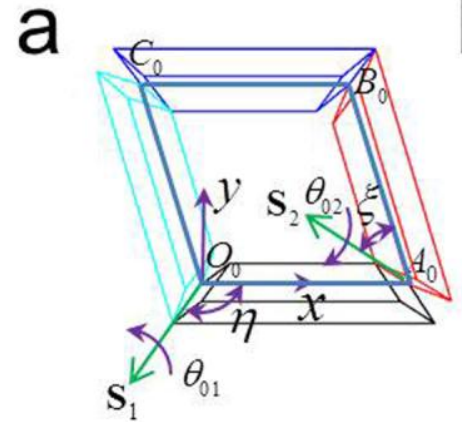

b

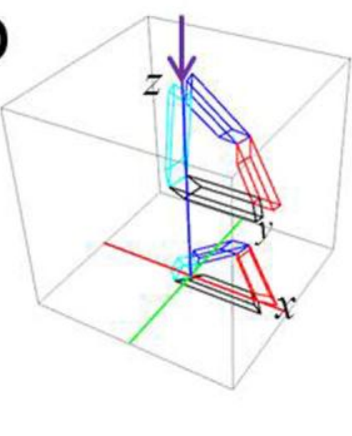

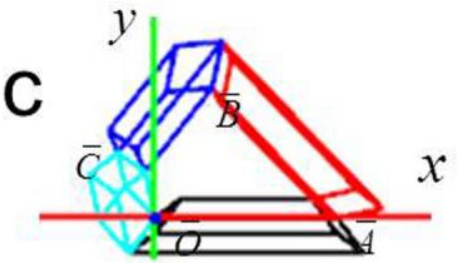

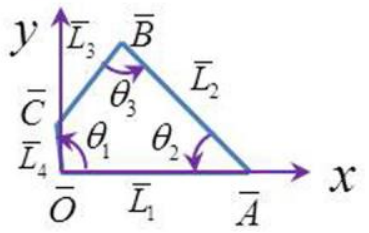

Fig. 12. Projection kinematics analysis of the Bennett linkage. ( $a, b$ and $c$ ) are the coordinate frame, sketch of the projection and projected configuration of the Bennett linkage respectively.

We previously reported the design of the DNA origami Bennett linkage [7,13]. Its dimensions are shown in Fig. A3. In addition, Fig. 12a shows the sketch of the Bennett linkage, the coordinate system and the rotation angles. Here, the initial configuration is determined when all of the points $\mathbf{O}_{0}, \mathbf{A}_{0}, \mathbf{B}_{0}$ and $\mathbf{C}_{0}$ are in the same plane and this plane is chosen as the $x y$ plane of the global coordinate frame. The lengths of the four edges are equal to $L$. The same as previous examples, $\alpha$ and $\beta$ are the global rotation angles used to determine the pose of the Bennett linkage in apace (Fig. 12b). Because it is much easier to measure joint angles than link lengths on TEM images, measuring the former is much more accurate than the latter. Here we only tested case 3 discussed in section 3.1, whose projection kinematics equations are based on three angles. Fig. 12c shows the detailed projected configuration of the Bennett linkage in 2D and the three angles, $\bar{\theta}_{1}, \bar{\theta}_{2}$, and $\bar{\theta}_{3}$ that can be obtained from the quadrilateral determined by the inner boundaries (bottom of Fig. 12c). The link lengths $\bar{L}_{1}, \bar{L}_{2}, \bar{L}_{3}$ and $\bar{L}_{4}$ were used to verify the solutions. The kinematics equations of the Bennett linkage were discussed in Table 11.

Table 11 Initial, rotated, projected and measured points that define the structure of DNA origami Bennett linkage

\begin{tabular}{|l|l|l|l|}
\hline Initial points & $\begin{array}{l}\text { Points after } \\
\text { rotation }\end{array}$ & Projected points & Measured points \\
\hline $\mathbf{A}_{0}=(L, 0,0)^{T}$ & $\mathbf{A}=[R] \mathbf{A}_{0}$ & $\begin{array}{l}\overline{\mathbf{A}}=[P] \mathbf{A} \\
=\left(A_{x}, 0,0\right)^{T}\end{array}$ & $\overline{\mathbf{A}}=\left(\bar{L}_{1}, 0,0\right)^{T}$ \\
\hline $\mathbf{B}_{0}=\mathbf{A}_{0}+\left[e^{\theta_{02} \mathbf{s}_{2}}\right]\left\{\begin{array}{c}-L \\
0 \\
0\end{array}\right\}$ & $\mathbf{B}=[R] \mathbf{B}_{0}$ & $\begin{array}{l}\overline{\mathbf{B}}=[P] \mathbf{B} \\
=\left(B_{x}, B_{y}, 0\right)^{T}\end{array}$ & $\begin{array}{l}\overline{\mathbf{B}}=\overline{\mathbf{A}}+ \\
\left(\bar{L}_{2} \cos \left(\pi-\bar{\theta}_{2}\right), \bar{L}_{2} \sin \left(\pi-\bar{\theta}_{2}\right), 0\right)^{T}\end{array}$ \\
\hline $\mathbf{C}_{0}=\left[e^{\theta_{01} \mathbf{s}_{1}}\right]\left\{\begin{array}{l}L \\
0 \\
0\end{array}\right\}$ & $\mathbf{C}=[R] \mathbf{C}_{0}$ & $\begin{array}{l}\overline{\mathbf{C}}=[P] \mathbf{C} \\
=\left(C_{x}, C_{y}, 0\right)^{T}\end{array}$ & $\overline{\mathbf{C}}=\left(\bar{L}_{4} \cos \bar{\theta}_{1}, \bar{L}_{4} \sin \bar{\theta}_{1}, 0\right)^{T}$ \\
\hline
\end{tabular}

Note: $A_{x}, B_{x}, B_{y}, C_{x}$ and $C_{y}$ were used to represent the coordinates elements because the explicit expressions of them are too complex. 
Again, equating the coordinates of projected to measured points in Table 11 yields five equations

$$
A_{x}=\bar{L}_{1}, B_{x}=\bar{L}_{1}+\bar{L}_{2} \cos \left(\pi-\bar{\theta}_{2}\right), B_{y}=\bar{L}_{2} \sin \left(\pi-\bar{\theta}_{2}\right), C_{x}=\bar{L}_{4} \cos \bar{\theta}_{1}, C_{y}=\bar{L}_{4} \sin \bar{\theta}_{1}
$$

The geometric constraint equation is given by the length of top blue link

$$
\left(\mathbf{B}_{0}-\mathbf{C}_{0}\right) \bullet\left(\mathbf{B}_{0}-\mathbf{C}_{0}\right)-L^{2}=0
$$

and $\theta_{02}$ can be solved from this equation when $\theta_{01}$ is given.

Similar to the case of planar four-bar linkages, the closed loop of the projected configuration introduces two additional constraint equations Eqs. $(17,18)$. Together with Eqs. $(31,32)$, eight equations were obtained. $\bar{L}_{1}, \bar{L}_{2}, \bar{L}_{3}$ and $\bar{L}_{4}$ were eliminated first, then the left four equations were the minimum equations used to solve for $\theta_{01}, \theta_{02}, \alpha$ and $\beta$.
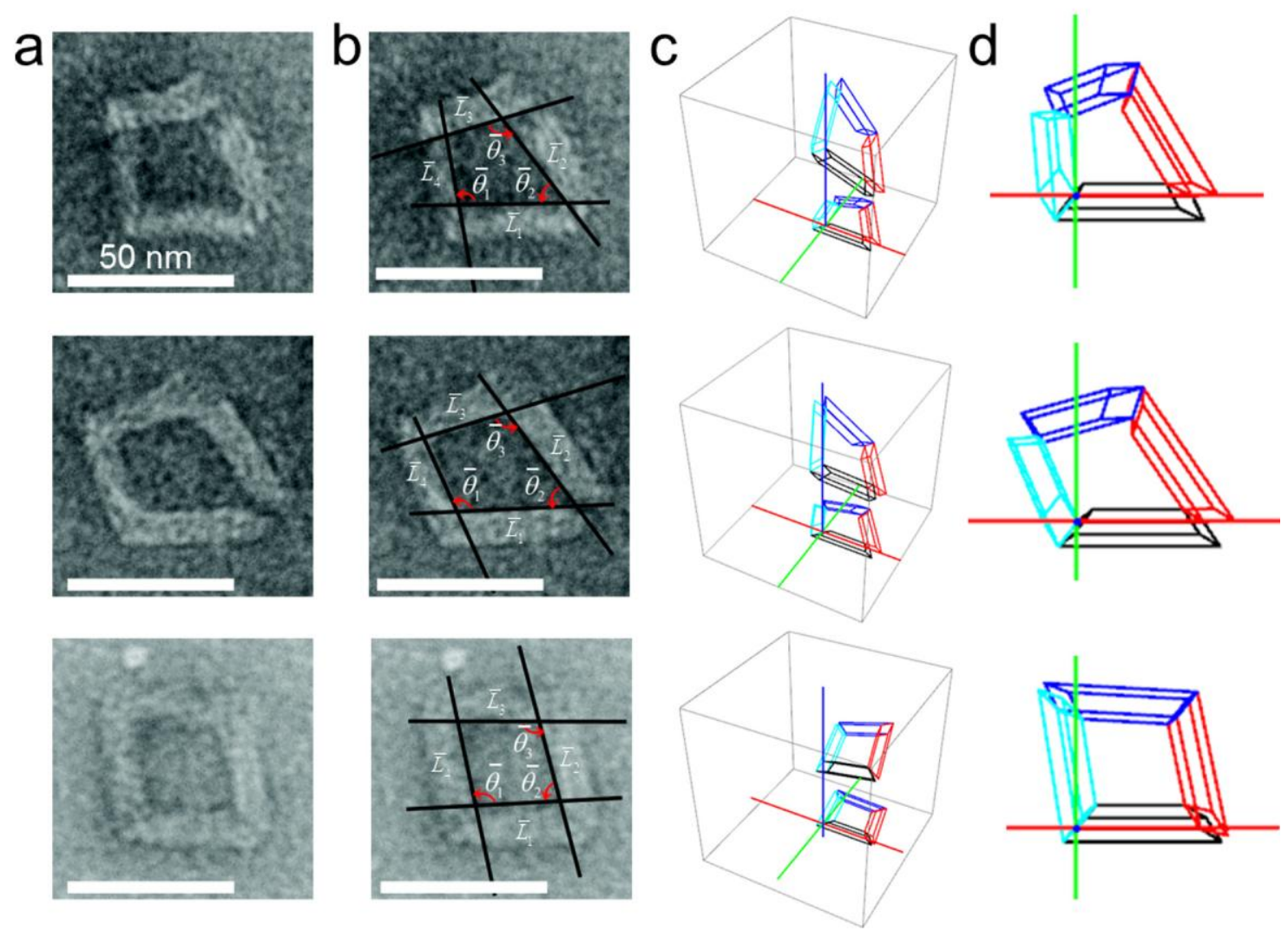

Fig. 13. Analysis of the projection kinematics of the Bennett linkage based on the TEM image. (a) Typical TEM images of the Bennett linkage, (b) projection information measured from the TEM image, (c) 3D projection solution of the TEM image sample, (d) detailed projected configuration of the solution.

The experimental analysis of the Bennett linkage fabricated by scaffolded DNA origami was described in [7]. Here three different TEM images of the Bennett linkage were picked, and each of them correspond to an individual Bennett linkage (Fig. 13a). Seven variables, $\bar{\theta}_{1}, \bar{\theta}_{2}, \bar{\theta}_{3}, \bar{L}_{1}, \bar{L}_{2}, \bar{L}_{3}$ and $\bar{L}_{4}$ were measured from each TEM images (Fig. 13b and Appendix material Table A3), and the measured three angles were used to solve the projection kinematics equations, and the measured link lengths were used to verify the solutions and only the one that can produce the minimum error between the derived projection link 
lengths and the measurements can be kept. The second column of the Appendix material Table A3 shows the obtained solution and the corresponding spatial and projection configuration are shown in Fig. 13c,d. The errors between the link lengths of the three TEM images and projected configurations derived from the solutions are $15.9 \%, 21.7 \%$ and $4.8 \%$ respectively (Column 4 of Table A3).

\section{Conclusions}

In this paper, we introduce the concept of the projection kinematics for identifying the possible configurations of mechanisms by only analyzing only one projected image. The motivation of this research comes from the need for the verification of the kinematics of DNA origami mechanisms (DOM). These DOM are self-assembled by programmed DNA base paring and function much like macroscopic mechanisms. We start with the projection kinematics analysis of revolute, prismatic, cylindrical and spherical joints and then extend it to general planar mechanisms. For planar mechanisms, we can always determine the unique configuration if the rotation directions of the joint can be distinguished from the projection image. For analysis of spatial linkages and application to real world DNA origami mechanism samples, we study universal joints and the Bennett four-bar linkage. We conclude that the reconstructed configuration is close enough to the original mechanism because they follow the kinematics constraints fairly well. Moreover, unique solutions may be obtained if two images taken from different directions are used for the projection kinematics analysis since the correct solution must be the common solution of the analyses based on the two projection images. Finally, the existence of multiple solutions suggests that it is important to design extra feature on the structures to eliminate the ambiguity.

\section{Acknowledgments}

This research is supported by the National Science Foundation under Grant No: CMMI-1235060, CMMI1536862. We also thank the Campus Microscopy and Imaging Facility (CMIF) of Ohio State University. Any opinions, findings, and conclusions or recommendations expressed in this material are those of the author(s) and do not necessarily reflect the views of the funding agencies.

\section{References}

[1] Seeman, N. C., 1982, "Nucleic acid junctions and lattices," J. Theor. Biol., 99, pp. 237-247.

[2] Seeman, N. C., 2010, "Nanomaterials based on DNA.," Annu. Rev. Biochem., 79, pp. 65-87.

[3] Watson, J. D., and Crick, F. H., 1953, "Molecular structure of nucleic acids," Nature, 171, pp. 737-738.

[4] Rothemund, P. W. K., 2006, "Folding DNA to create nanoscale shapes and patterns," Nature, 440(7082), pp. $297-302$.

[5] Castro, C. E., Kilchherr, F., Kim, D.-N., Shiao, E. L., Wauer, T., Wortmann, P., Bathe, M., and Dietz, H., 2011, “A primer to scaffolded DNA origami," Nat. methods, 8(3), pp. 221-229.

[6] Douglas, S. M., Dietz, H., Liedl, T., Högberg, B., Graf, F., and Shih, W. M., 2009, "Self-assembly of DNA into nanoscale three-dimensional shapes," Nature, 459(7245), pp. 414-418.

[7] Marras, A. E., Zhou, L., Su, H.-J., and Castro, C. E., 2015, "Programmable motion of DNA origami mechanisms," Proc. Natl. Acad. Sci., 112(3), pp. 713-718.

[8] Castro, C. E., Su, H.-J., Marras, A. E., Zhou, L., and Johnson, J., 2015, "Mechanical design of DNA nanostructures," Nanoscale 7, pp. 5913-5921.

[9] Marras, A. E., Zhou, L., Kolliopoulos, V., Su, H. J., and Castro, C. E., 2016, "Directing folding pathways for multicomponent DNA origami nanostructures with complex topology," New J. Phys., 18(5), pp. 055005/1-055005/9.

[10] Zhou, L., Marras, A. E., Su, H.-J., and Castro, C. E., 2013, "DNA Origami Compliant Nanostructures with Tunable Mechanical Properties," ACS nano, 8(1), pp. 27-34.

[11] Zhou, L., Marras, A. E., Su, H.-J., and Castro, C. E., 2015, “Direct Design of an Energy Landscape with Bistable DNA Origami Mechanisms," Nano Lett., 15(3), pp. 1815-1821.

[12] Zhou, L., Marras, A. E., Castro, C. E., and Su, H.-J., 2016, "Pseudorigid-Body Models of Compliant DNA Origami Mechanisms," J. Mech. Robot., 8(5), pp. 051013/1-051013/11.

[13] Su, H.-J., Castro, C. E., Marras, A. E., and Hudoba, M., 2012, "Design and Fabrication of DNA Origami Mechanisms 
and Machines," Advances in Reconfigurable Mechanisms and Robots I, Springer London, pp. 487-500.

[14] Sun, B., Edgar, M. P., Bowman, R., Vittert, L. E., Welsh, S., Bowman, A., and Padgett, M. J., 2013, “3D

Computational Imaging with Single-Pixel Detectors," Science, 340(6134), pp. 844-847.

[15] Zhang, Y., Gibson, G. M., Hay, R., Bowman, R. W., Padgett, M. J., and Edgar, M. P., 2015, “A fast 3D reconstruction system with a low-cost camera accessory," Sci. Reports, 5(10909), pp. 10909/1-10909/7.

[16] Marton, Z. C., Pangercic, D., Blodow, N., Kleinehellefort, J., and Beetz, M., 2010, "General 3D modelling of novel objects from a single view," Intelligent Robots and Systems (IROS), 2010 IEEE/RSJ International Conference on, pp. 3700-3705. [17] Barazzetti, L., Scaioni, M., and Remondino, F., 2010, "Orientation and 3D modelling from markerless terrestrial images: combining accuracy with automation,” Photogramm. Rec., 25(132), pp. 356-381.

[18] Bartesaghi, A., Merk, A., Banerjee, S., Matthies, D., Wu, X., Milne, J. L., and Subramaniam, S., 2015 , “2.2 $\AA$ resolution cryo-EM structure of $\beta$-galactosidase in complex with a cell-permeant inhibitor," Science, 348(6239), pp. 1147-1151.

[19] Zhang, L., Lei, D., Smith, J. M., Zhang, M., Tong, H., Zhang, X., Lu, Z., Liu, J., Alivisatos, A. P., and Ren, G., 2016, "Three-dimensional structural dynamics and fluctuations of DNA-nanogold conjugates by individual-particle electron tomography," Nat. Commun., 7(11083), pp. 11083/1-11083/10.

[20] Bai, X., Martin, T. G., Scheres, S. H., and Dietz, H., 2012, “Cryo-EM structure of a 3D DNA-origami object,” Proc. Natl. Acad. Sci., 109(49), pp. 20012-20017.

[21] Ruf, A., and Horaud, R., 1999, "Visual servoing of robot manipulators part I: Projective kinematics,” Int. J. Robot. Res., 18(11), pp. 1101-1118.

[22] Jin, H., Wu, F., Yang, C., Chen, L., and Li, S., 2014, "A Review of 3-D Reconstruction Based on Machine Vision," Intelligent Robotics and Applications: 7th International Conference, ICIRA 2014, Guangzhou, China, December 17-20, 2014, Proceedings, Part II, Springer International Publishing, Cham, pp. 195-203.

[23] Sonka, M., Boyle, R., and Hlavac, V., Image Processing: Analysis and Machine Vision, PWS Pub., Pacific Grove, CA, 1999.

[24] Verschelde, J., 2011, "Polynomial homotopy continuation with PHCpack," ACM Commun. Comput. Algebra, 44(4), pp. 217-220.

[25] Schindelin, J., Rueden, C. T., Hiner, M. C., and Eliceiri, K. W., 2015, “The ImageJ ecosystem: An open platform for biomedical image analysis," Mol. Reprod. Dev., 82, pp. 518-529. 
Measure projected geometric parameters for a 2D TEM image

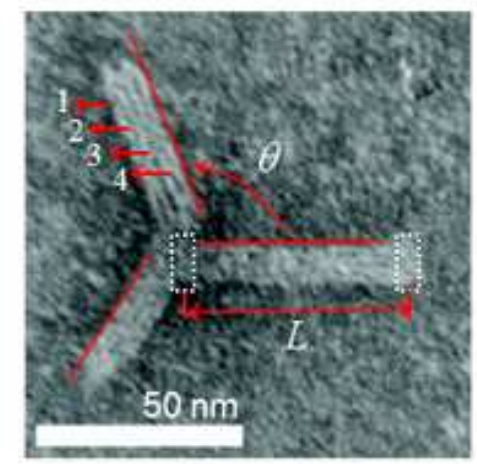

Projection Kinematics

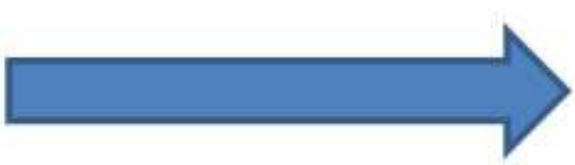

Projection Kinematics

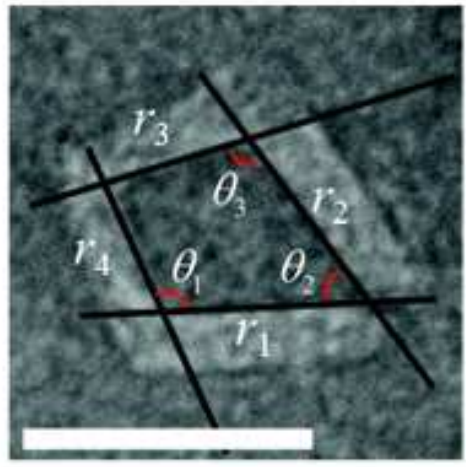

Reconstruct 3D Configuration of DNA Origami Mechanisms

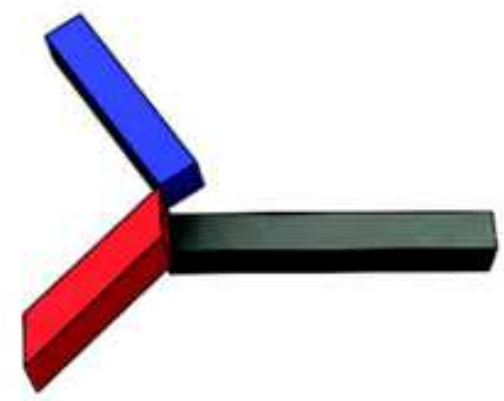

Universal Joint

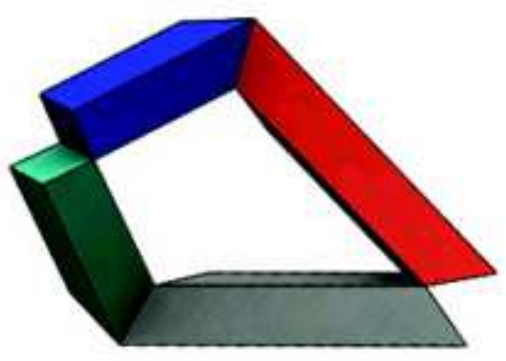

Bennett 4-bar Linkage 